\title{
Cognitive Radio in Digital Agriculture: A Physical Layer Overlay Based Underground Radio Propagation System
}

\section{Usman Raza}

Purdue University https://orcid.org/0000-0002-2274-3783

Abdul Salam ( $\sim$ salama@purdue.edu )

Purdue University

Umit Karabiyik

Purdue University

\section{Research}

Keywords: Cognitive radios, Radio spectrum management, Wireless networks, Load Balancing

Posted Date: November 17th, 2020

DOl: https://doi.org/10.21203/rs.3.rs-106014/v1

License: (c) (1) This work is licensed under a Creative Commons Attribution 4.0 International License.

Read Full License 
Noname manuscript No.

(will be inserted by the editor)

\title{
Cognitive Radio in Digital Agriculture: A Physical Layer Overlay Based Underground Radio Propagation System
}

\author{
Usman Raza • Abdul Salam • Umit \\ Karabiyik \\ Received: date / Accepted: date
}

\begin{abstract}
In digital agriculture, the cognitive radio technology is being envisaged as solution to spectral shortage problems by allowing agricultural cognitive users to co-exist with PU in the same spectrum on the field. Cognitive radios increase system capacity and spectral efficiency by sensing the spectrum and adapting the transmission parameters. This design requires a robust, adaptable and flexible physical layer to support cognitive radio functionality. In this paper, two different approaches are proposed to improve the application of cognitive radios in agriculture. A novel physical layer architecture for cognitive radio based on cognition, cooperation, and cognitive interference avoidance has been developed by using power control for digital agriculture applications. The design is based on sensing of spectrum usage, detecting the message/spreading code of PU, cognitive relaying, cooperation, and cognition of channel parameters. Moreover, the power and rate allocation, ergodic, and outage capacity formulas are also presented. Furthermore, a two-hop load balancing approach is presented using a cognitive radios as a relay node in the network to improve throughput and optimize the load between different area of an agricultural field.
\end{abstract}

Keywords Cognitive radios · Radio spectrum management · Wireless networks · Load Balancing

U. Raza

Purdue University, USA.

E-mail: uraza@purdue.edu

A. Salam (Corresponding Author)

Purdue University, USA.

E-mail: salama@purdue.edu

U. Karabiyik

Purdue University, USA.

E-mail: umit@purdue.edu 


\section{Introduction}

United Nations has reported an increase of $33 \%$ population by 2050 which will also result in sudden increase in food demand [9]. Agricultural sector meets the world's food demand, hence, progress of agriculture is associated with the economic and social growth of the world. Therefore, with limited available resources, it is very important to improve the efficiency of this sector to cope up with the food demand of increasing population. Digital agriculture aims to solve this issue by digitizing the agricultural sector by employing sensing and communication technology to gather various soil and crop properties from the field to central computer servers for decision making purposes. The main purpose is to modernize and improve agricultural activities such as soil monitoring and irrigation etc., to minimize losses [24]. Wireless systems are mostly deployed in the agricultural fields for the communication. The reason is because of agricultural fields are mostly cultivated in the remote areas, it is very difficult to employ wired networking solutions for communication purposes. Moreover, with various mobile equipment such as tractors and harvests etc., wireless solutions are most suited for communication in agriculture sector.

Wireless communication uses radios waves to transmit data. Radio, being a broadcast medium, is prone to interfaces from the users utilizing the same frequency band. With ever increasing wireless applications, there is a shortage of free wireless spectrum for new wireless applications and systems [17]. Cognitive radios are considered enabling technology to solve this spectrum shortage problem. It employs advanced signal and radio technologies along-with the spectrum allocation policies for new users. In this area, the cognitive radio operation holds the promise for flexible, inexpensive radio devices with dynamic spectrum management techniques for digital agriculture sensing and communication applications [40]. This technology can fill the gaps in on-field radio spectrum and can also increase spectral efficiency through sensing of wireless spectrum and adaptive communications $[5,10,11,15,18,21,22,25,26,29,30,43,50-53]$.

The Federal Communication Chart (Federal Communication Chart (FCC)) has permitted the use the cognitive radio devices in the spectrum range of $470 \mathrm{MHz}$ to $698 \mathrm{MHz}$ on farm machinery and agricultural equipment for digital agriculture applications [35-38].In 2008, FCC already had allowed the operation of unlicensed cognitive devices in UHF TV band [14]. In 2010, restriction of mandatory sensing requirements was removed [8] which has facilitated the use of the spectrum with relocation-based channel allocation. Non-cognitive users are also known as Primary Users (Primary Users (PU)) because they are the incumbent licensed users. Similarly, cognitive unlicensed users are also known as secondary users (Secondary Users (SU)) and flexibly share the spectrum with PUs. These terms are used interchangeably in this paper.

Three paradigms namely: underlay, overlay, and interweave, are used for cognitive radio implementation [17]. Underlay paradigm does not allow a simultaneous transmission of PU and SU unless the interference due to SU at $\mathrm{PU}$ transmitter is under some reasonable and accepted threshold. Interweave 
paradigm uses the fact that major portion of the spectrum is not always being fully utilized. Hence, an Interweave cognitive radio can sense the underutilized spectrum and opportunistically communicates in that spectrum causing minimal interference to PUs. In overlay paradigm, the cognitive user is aware of the code-book and message of PU through some pre-decided mechanism, e.g., periodic broadcast by PUs. Overlay paradigm allows the simultaneous transmission of PUs and SUs. Cognitive transmitter's knowledge of message/code is used by PU to cancel the interference of peer PUs. It also helps with the PU data transmission by allocating some portion of cognitive power to further relay the PU transmission. This trade-off increases the signal-to-interference and noise ratio (Signal-to-Interference and Noise Ratio (SINR)) of PU through relaying viz-a-viz decrease in SINR caused by interference of other cognitive users. It also helps in keeping rate of PU unaffected.

Moreover, SUs and PUs operate using a shared spectrum and wireless channels. To that end, SU must use the spectrum in such a way that it does not cause a significant interference to PUs. Therefore, it is important to devise an efficient mechanism of resources sharing between PUs and SUs. To that end, a load balancing algorithm is required between primary and secondary users [7]. This paper considers centralized architecture where a multiple base stations are controlling a set of SUs. Such centralized architecture is also used in [12].

The paper can be divided into two independent sections to improve the operation of cognitive radios in agriculture. The first section aims to improve architecture of cognitive radios in agriculture. First section presents a Cognitive Direct Sequence Spread Spectrum (Cognitive Direct Sequence Spread Spectrum (CDSSS)), a cooperative overlay approach at the physical layer of cognitive radio in smart agriculture. CDSSS can be utilized for white space communications on the field. The potential of CDSSS as an overlay cognitive radio. In this collaborative protocol, the cognitive users exchange message information that is used in synchronization and improving knowledge of presence of primary users. The Multi-user detection (MUD) is employed at cognitive receiver in order to reduce multiple access interference and intersymbol-interference. The capacity region, merits, and challenges of CDSSS are also discussed.

Second section of the paper attempts to improve application of cognitive radios in agriculture. It improves quality of service by maximizing resource allocations and spectrum sharing between the SUs and PUs using a centralized base-station based approach. The basic idea is to divide an agricultural field into multiple clusters with each cluster having a base station of its own. Every base station shares its state with neighboring base stations. If any of the base station is overloaded, it relays its traffic to underloaded base station. The intuition is to use each base station to its full extent while relaxing the load of any overloaded base station. This approach improves the average throughput of the system and balances the traffic load between the clusters.

This paper is organized as follow: Section 2 to 6 presents first part of paper with related work discussed in Section 2, system model presented in Section 3, 
the adaptive power and rate control are presented in Section 4 . Finally, results of the developed overlay approach presented in Section 5 and future work in Section 6. Moreover, Section 7 to 8 presents second part of paper with related work discussed in Section 7 and proposed methodology in Section 8. Finally, the paper is concluded in Section 9.

\section{Cognitive Direct Sequence Spread Spectrum (CDSSS)}

\section{A Related Work}

Cognitive radio has attracted a lot of research focus since its inception in 2000 [31]. Cognitive radio is a software defined radio with dynamic frequency, modulation type, and transmitted power configuration [20]. The IEEE 802.22 work group: Wireless regional Area Networking Work Group (Wireless Region Area Networking (WRAN)), was created in 2004. Its purpose was to define physical (PHY) and data layer (Media Access (MAC)) standards [1], and to develop standards for cognitive devices to be used in TV spectrum. To achieve co-existence with existing services, it uses spectrum sensing, licensed user detection, and spectrum management techniques.

The physical layer design issues unique to cognitive radio systems which can deteriorate the performance of cognitive radio are discussed in [3]. It indicates that the critical design problem related to cognitive receiver is to meet tight requirements on radio sensitivity and detection of weak signals with restricted dynamic range. In [34], interference, coordination and cooperation have been discussed as fundamental design trade-offs in cognitive radio systems. In [46], author investigates security at physical layer of cognitive radios in non-orthogonal multiple access (Non-Orthogonal Multiple Access (NOMA)) networks. Author in [2] propose Dynamic spectrum access (Dynamic Spectrum Access (DSA)) to use the existing spectrum without any interference. They use device fingerprinting to detect any change in bandwidth of the non-cognitive device in the spectrum. In [32], a strategy is formulated for non-cognitive user selection based on dynamic game pricing approach. In [47] an opportunistic spectrum access scheme has been proposed which imposes restriction on cognitive user transmission power to avoid interference to PU.

To the best of our knowledge, proposed manuscript paper is the first design to consider MultiUser Detection (MUD) at the transmitter level. It results in enhancement of signal quality of non-cognitive user and also compensate the interference that impacts the cognitive user. This novel architecture can be employed in ad-hoc wireless networks and Internet of Underground Things due to its cognitive and cooperative nature [44].

\section{Methodology}

The CDSSS operates in asynchronous fading inter-user channel. It uses nonorthogonal spreading codes that results in multiple access interference and 


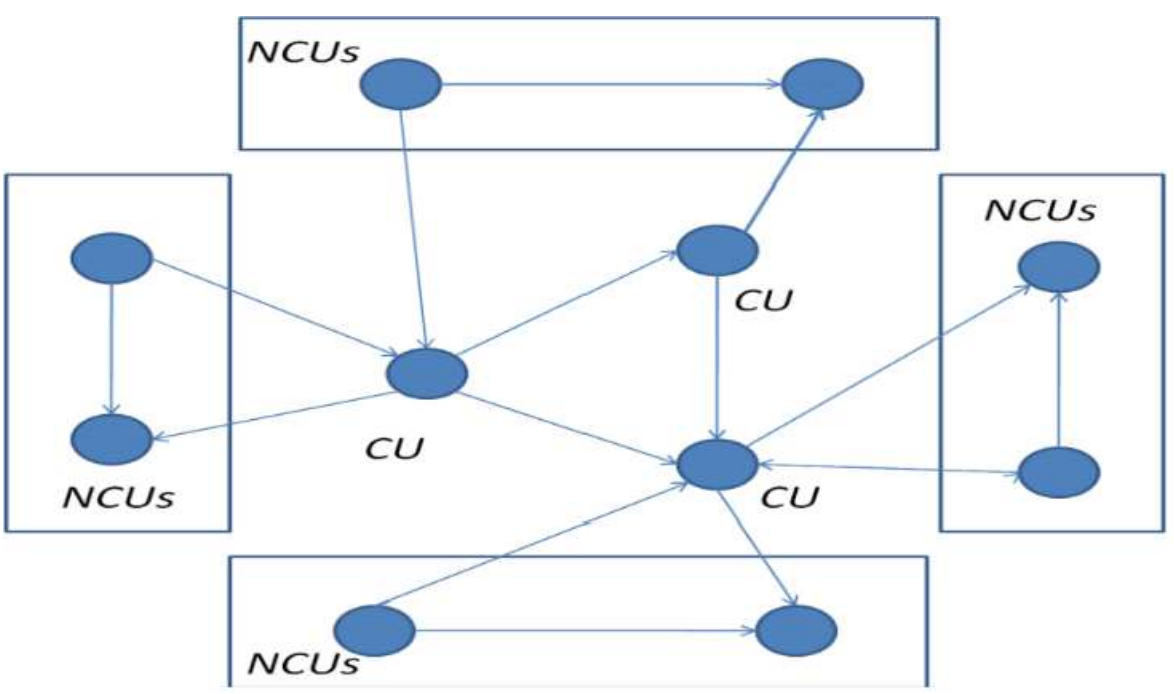

Fig. 1. The interaction among cognitive radios and PUs

inter-symbol interference. Orthogonal codes restrict the number of users that system can support due to which non-orthogonal spreading codes are used. Different techniques (e.g., hybrid spreading sequences [4,19,42], multiple spreading sequences [27], and quasi orthogonal spreading sequences [6,13]) exists in literature to increase the capacity and to accommodate higher number of users. Hybrid concept is based on augmenting orthogonal codes with non-orthogonal codes. Multiple spreading codes concept uses two set of orthogonal codes. In this work, the non-orthogonal codes are employed. These codes do not satisfy the cross-correlation property.

In CDSSS, cognitive users share the spectrum simultaneously with PU by adapting the transmit power to keep the interference caused to PU below the noise floor of the spectrum. We assume that the cognitive users are spatially scattered according to a homogeneous Poisson point process. The power control mechanism for allocation of power to cognitive users has been developed based on interference, spectrum utilization, and the number of active PUs. In the design, transmit power can be adjusted flexibly in a short time span. The mandatory constant spectrum sensing for the transmit power adaption is enforced to mitigate interference to primary users during longer transmission windows of cognitive users. SUs uses the spectrum sensing mechanism to detect information about PUs such as their spectrum utilization and activation. Therefore, based on this knowledge, it adjusts its transmit power accordingly.

In the CDSSS design, the PUs are unaware of presence of cognitive users in the near vicinity. However, the cognitive users have the ability to facilitate the primary user transmission through relaying. On activation, cognitive users sense spectrum and detect PU. On detection of a non-cognitive user, it adapts the transmit power accordingly, relay message of non-cognitive user, and ex- 


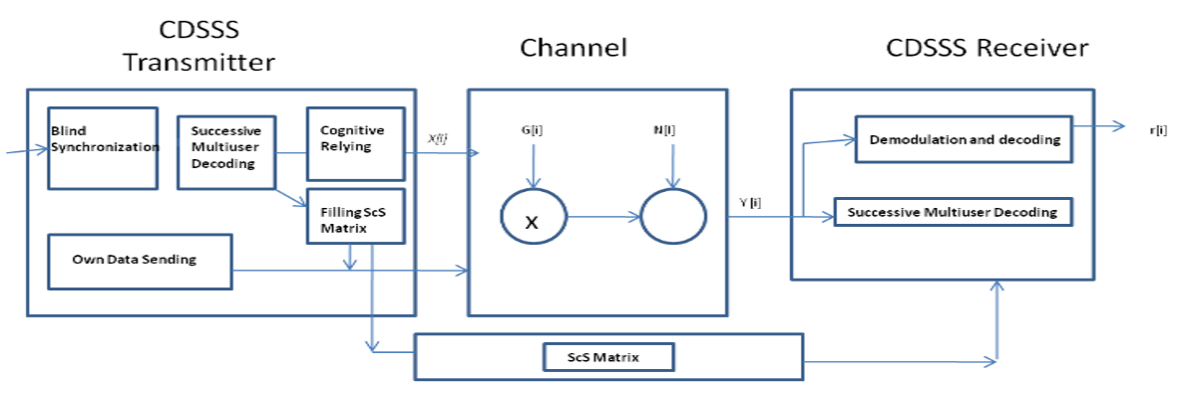

Fig. 2. The CDSSS system model

change the detected information with other cognitive users. Other cognitive users also attempt to detect the same information concurrently. This combined cognition/cooperation helps in maintaining the accurate and updated information about primary users and also facilitates synchronization. In the last step, it sends its own message with delay to destination non-cognitive user. When non-cognitive user is not detected, cognitive user does not adapt the transmit power and can proceed to send its own message without waiting.

In Fig. 1, an interaction among cognitive users (represented as CU) and PUs (represented as NCU, i.e., non-cognitive users) is shown. Suppose D be the set of PU and $\mathrm{C}$ be the set of cognitive users. Let $\mathrm{L} \in \mathrm{C}$ be the set of relaying cognitive users that decode and forward messages of PU. In phase 1,PU in set $D=D_{1}, D_{2}, D_{3}, \ldots . D_{N}$ transmits their symbol $s_{l}$. The CDSSS transmitter works in two steps: a cognition step, which includes blind synchronization and decoding. By cognitively relaying the message of PU, in cooperation stage, the detected information about PU is exchanged with other cognitive users. Second step also includes sending of own data by CDSSS transmitter. The CDSSS system model is shown in Fig. 2. These steps of CDSSS transmitter are individually discussed in the following sub-sections.

\section{A Cognition: Decoding and Cognitive Relaying}

In CDSSS, synchronization is performed by using the blind synchronization process that works without any prior knowledge of cognitive and non-cognitive transmitters. By this method of cognition, knowledge of spreading sequences is acquired. Cognitive users who cannot perform decoding acquires this knowledge through cognition process (explained in sub-section 3.B). A knowledge of spreading sequences is required for correlation in the Successive Multi-user Decoding (Successive Multi-user Decoding (SMD)) and for relaying. The CDSSS transmitter performs detection after synchronization. As an asynchronous channel is assumed, hence, unlike synchronous channel where detection can be done by focusing on one-bit interval, there is an overlap in different bit intervals. The detection process considers overlapping bits which consequently 
lead to formulation of detection problem over the whole message [29]. The received signal at cognitive transmitter can be written as:

$$
r(t)=\sum_{k=1}^{K} A_{k} g_{k}\left(t-\tau_{k}\right) d_{k}\left(t-\tau_{k}\right)+n(t)
$$

where $A_{k}(t), g_{k}(t)$ and $d_{k}(t)$ are the amplitude, signature code form and modulation of $k-t h$ user, respectively, $\tau_{k}$ is delay for user $k$ and $n(t)$ is additive white Gaussian noise.

The SMD takes a serial approach for detecting and decoding multiple users. SMD works in multiple stages. In every stage, SMD selects a user to decode in ascending order of received power, and decode by using correlation matrix $\mathrm{R}$ which is populated with spreading codes through cooperation and cognition. The process of information distribution and own data sending by a cognitive radio is explained in sub-section 3.B.

The output of the first stage of SMD gives data of cognitive user 1 and a modified received signal without non-cognitive user 1 . This signal then becomes input to next stage, that repeats process of stage 1 for rest of the PU. The strongest power user is selected first in SMD because of ease of achieving acquisition and demodulation This multi-user decoding process can be implemented in parallel, where all the PU can sense in parallel at the cost of additional hardware. Assuming perfect amplitude and delay estimation, the received signal for non-cognitive user $k$ is given as:

$r\left(t-T_{b}\right)=\sum_{k=1}^{K}\left(d_{k}\left(t-\tau_{k}-T_{b}\right)\left(A_{k}\left(t-\tau_{k}-T_{b}\right)\right) * g_{k}\left(t-\tau_{k}-T_{b}\right)\right)+n\left(t-T_{b}\right)$

where $T_{b}$ is the time delay. After every decoding, decision variable of the next user under decoding is affected by multiple access interference of remaining users, Gaussian noise, and cumulative noise due to some imperfect decoding. The Gaussian approximations can be used to calculate the bit error rate (Bit Error Rate (BER)) of SMD while assuming Gaussian noise with zero mean. The probability of bit error after $j_{t h}$ decoding, conditioned on the amplitude, can be expressed as $Q$ function.

SMD requires simple multipliers and adders. The delay of the SMD is limited by the performance of the correlators. As decoding is done in the successive manner, the maximum number of decoding by a cognitive user is limited by the speed of performing correlation. In order to ensure the flow of symbols at the symbol rate Rs, the speed of correlator must be N.Rs, where N is the possible number of decoding. For example, in order to have at least 110 decoding assuming a bit rate of $10 \mathrm{~kb} / \mathrm{s}$, the speed of the correlator must be at least $0.17 \mathrm{MHz}$ (i.e. each correlator take less than 6.50 micro seconds). Thus, processing speed of the hardware may limit the number of possible decoding.

Other limiting factor is the number of correlators (matched filters) required for SMD front end in CDSSS transmitter (Fig. 3). Usually number of active 


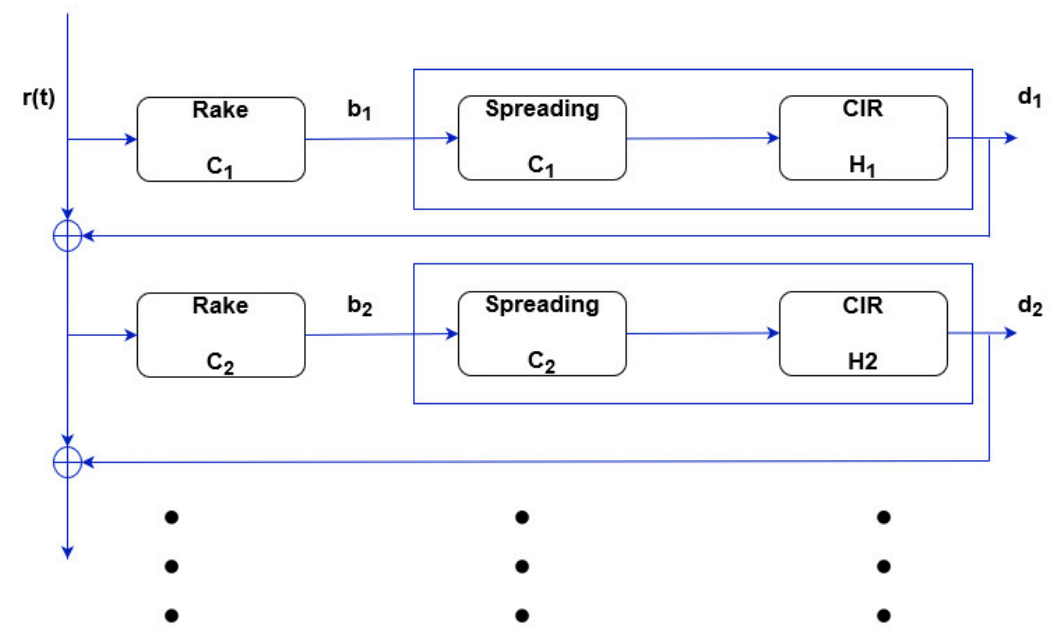

Fig. 3. Successive multi-user decoding

users is much less than total number of users. This number is further reduced in the vicinity of CDSSS transmitter performing cognition. Therefore, SMD correlates the received signal with a set of $\mathrm{N}$ correlating signals, where $\mathrm{N}$ may be dependent on the strength ranking of the user's received signal. Based on this ranking, a threshold can be defined for performing maximum correlations. Moreover, as only the SMD performs the cognition, hence, after performing the decoding up to threshold level, the remaining signal can be discarded without affecting the system performance.

In SMD, virtual multi-path created by the relays are exploited by employing the RAKE for collecting multi-path delayed by integer multiple of chip time. The RAKE also exploits the frequency diversity introduced by frequency selective fading and is placed before the correlator in the SMD. In CDSSS, cognitive users also serve as a relay for PU. Based on a full duplex radio operation, while the bits are being decoded, they are also passed to the transmitter for relaying purposes, i.e., decode and relay operations are being done simultaneously. Both operations are completed using the same spread code with concurrent population of the spread code vector.

A cooperative relay scheme for cognitive communication has been proposed in [18]. As an alternative to relaying same message of non-cognitive user, the relays use coded cooperation. In the code combining, the non-cognitive user transmits a code word to target non-cognitive radio and other cognitive radio helps the cognitive sender by sending additional redundancy bits. Accordingly, the non-cognitive receiver combines the original code word and redundancy bits to decode the source message. The coded diversity was introduced in $[41,43]$. Analog network coding (Analog Network Coding (ANC)), lattice, and dirty paper coding are other alternative techniques for coded cooperation. 
Other cognitive radios populate their spreading code vector $\mathrm{R}$ by the same cognition process that is explained above. However, the cooperation comes to play a role here for other cognitive users which are unable to decode the non-cognitive user due to fading or other phenomena. Information distribution process among cognitive radios through cooperation and own data sending is presented in the next section.

\section{B Cooperation: Information Distribution and Own Data Transmission}

The cognitive users in CDSSS scheme exchange spreading codes and knowledge of amplitudes/channel gains from PU to cognitive users through a novel collaborative protocol. Although each cognitive user has the value of channel gains for a particular non-cognitive user different from other cognitive users, it still helps in mapping general state of channel form cognitive to PU. Accordingly, that is used for indirect relaying based on channel state. This cooperation phase for information distribution among cognitive users is combined with own data sending. When CUs starts functioning, it decodes PU and populate its $R$. After that, it selects the spreading code from $R$ and use it to send its own data along with collaboration protocol explained below:

The cognitive network is a random geometric graph $G(C, R)$, where $C \operatorname{cog}$ nitive users are chosen uniformly and each pair of cognitive users is connected if their Euclidian distance is smaller than some transmission radius $R$, also called the connectivity radius.

1. For each cognitive user n, let $C(n)$ represents the set of neighbors of $n$.

2. User $n$ constructs the info exchange message based on the values of ScS vector.

3. This message is then combined with the own data sending.

4. Modulation and spreading process is performed.

5. This message is then broadcasted with 1-bit flag that indicates the message is meant for non-cognitive user.

6 . The broadcast value is successfully received by the nodes that are within the radius $R$.

7. All neighbors receive the broadcast value and update their $\mathrm{ScS}$ vector.

8. This procedure takes place at every cognition stage and terminates when all of $\mathrm{ScS}$ vector has been populated.

Cognitive radio receiver also employs successive multiuser decoding for decoding the desired message and also for subtracting multiple access interference.

\section{Cognitive User Rate and Power Control}

A power control scheme has been developed that provides protection to PU from cognitive users interference by maintaining their SINR above the required 
thresholds. Cognitive users are allowed to transmit data according to assigned power and rates. We derive cognitive power allocation strategies to achieve the ergodic and outage capacity under the defined non-cognitive user outage probability constraint [25].

Suppose $B$ is the bandwidth and $R_{c}$ is the data rate of cognitive radio. Let $P_{i}^{c}$ be the transmit power of the cognitive user and $P_{i}^{n}$ be the transmit power of the non-cognitive user. Let $G_{i j}^{n n}$ be the channel gain between two PU, $G_{i j}^{c c}$ the channel gain between to cognitive users, $G_{i j}^{c n}$ channel gain between cognitive user $i$ and non-cognitive user $j$ and $G_{i j}^{n c}$ be the channel gain between non-cognitive user $i$ and cognitive user $j$. Due to the presence of the cognitive users and the corresponding multiple access interference, we can formulate the SINR of the $i_{t h}$ non-cognitive user as:

$$
\gamma_{i}^{n}=\frac{P_{i}^{n} G_{i j}^{n n}+\rho \sum_{j=1}^{N} P_{j}^{c} G_{i j}^{c n}}{\rho \sum_{j=1}^{N} P_{j}^{c} G_{i j}^{c n}+N_{0}}
$$

where $N_{0}$ is the power spectral density of a constant background noise and $\rho$ is interference reduction due to processing gain. Second term in the numerator of equation (3) is the power of the cooperating cognitive user which improves the SINR of the non-cognitive user by relaying the data. $N^{\prime}$ represents all the relaying PU with ability to decode-and-forward message to PU.

The SINR of cognitive user is defined as:

$$
\gamma_{i}^{c}=\frac{P_{i}^{c} G_{i j}^{c c}}{\rho \sum_{j=1}^{N} P_{j}^{c} G_{j i}^{c n}+N_{0}}
$$

Outage probability for PU can be defined as $p_{\text {out }}^{n}=p\left(\gamma_{i}^{n}<\gamma_{i}^{t h}\right)$. The outage probability for cognitive users (SU) can be defined as $p_{\text {out }}^{c}=p\left(\gamma_{i}^{c}<\gamma_{i}^{0}\right)$. Ergodic capacity for cognitive users under non-cognitive constraint is:

$$
\begin{aligned}
\max \mathrm{E}\left\{\log _{2}\left(1+\gamma_{i}^{c}\right)\right\} \\
\text { such that } p_{\text {out }}^{n} \leq p_{\text {out }}^{c} \\
p_{i}^{c} \geq 0 \\
p_{i}^{c} \leq p_{\text {max }}^{c}
\end{aligned}
$$

Outage capacity for cognitive users under non-cognitive constraint is given as:

$$
\begin{aligned}
& \min \mathrm{p}\left\{\log _{2}\left(1+\gamma_{i}^{c}<R^{c}\right)\right\} \\
& \text { such that } p_{\text {out }}^{n} \leq p_{\text {out }}^{-c} \\
& p_{i}^{c} \geq 0 \\
& p_{i}^{c} \leq p_{\text {max }}^{c}
\end{aligned}
$$

where $R_{c}$ is the predefined constant rate cognitive radio. 


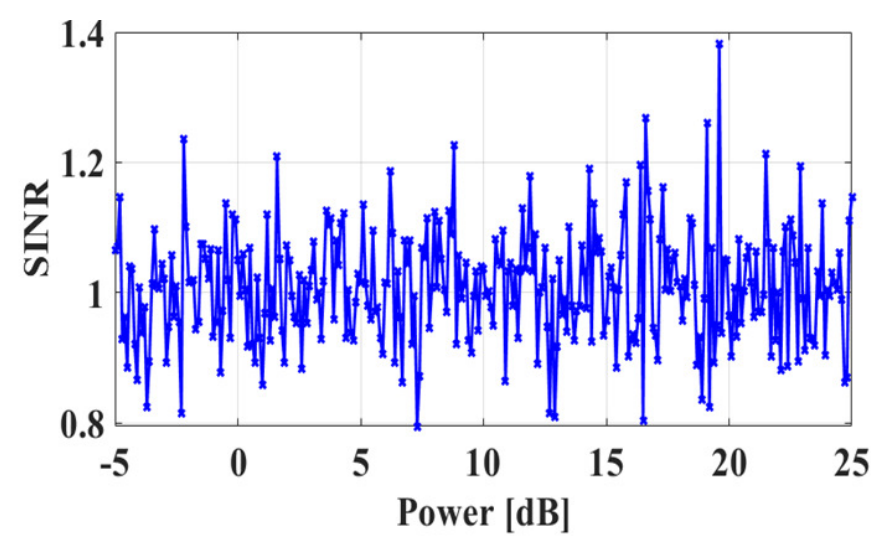

Fig. 4. SINR vs. Power $[\mathrm{dB}]$ : Non-cognitive user transmission is assisted by cognitive users

Under CDSSS power and rate optimization scheme can be formulated as follows:

$$
\begin{gathered}
\max \sum_{i \in C} \gamma_{i}^{c} \\
\text { such that } 0<P_{i}<P_{i}^{\max } \forall_{i \in C} \\
\gamma_{i}^{n} \geq \gamma_{i}^{T H}<\forall_{i \in I} \\
\gamma_{i}^{c} \geq \gamma_{0}^{c}<\forall_{i \in C}
\end{gathered}
$$

By solving equation (7), we get optimum SINR $\gamma_{i}^{c}$ for non-cognitive user. Substituting this resultant maximum SINR in equation (5) and (6), we get that ergodic and outage capacity of the CDSSS under outage constraint of non-cognitive user.

Here it should be noted that a cognitive user can increase its rate by increasing its power but in the process, it decreases the rate of other cognitive users due to multiple access interference it causes to them. Accordingly, by decreasing the power of a particular cognitive user, the date rate of other cognitive users is increased by reduction in multiple access interference.

\section{Results and Discussion}

In this section, we present the performance evaluation of the proposed approach. The CDSSS simulations are done using the MATLAB. Three cases are considered and in each case two scenarios are presented. In sub-section 5.A, the results for maximum cognitive cooperation case are presented. The limited cognitive cooperation case is discussed in sub-section 5.B. The third case of cognitive interference is evaluated in sub-section 5.C. 


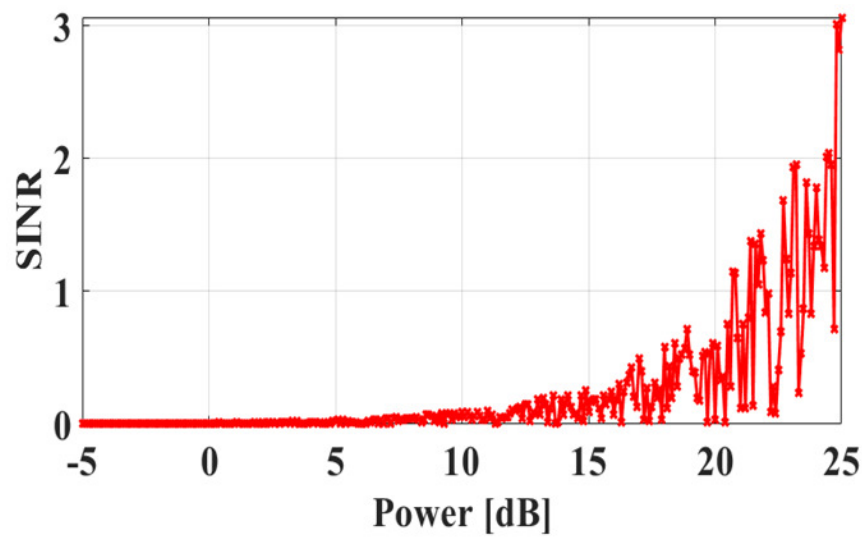

Fig. 5. SINR vs. Power $[\mathrm{dB}]$ : Non-cognitive user transmission with no-assistance

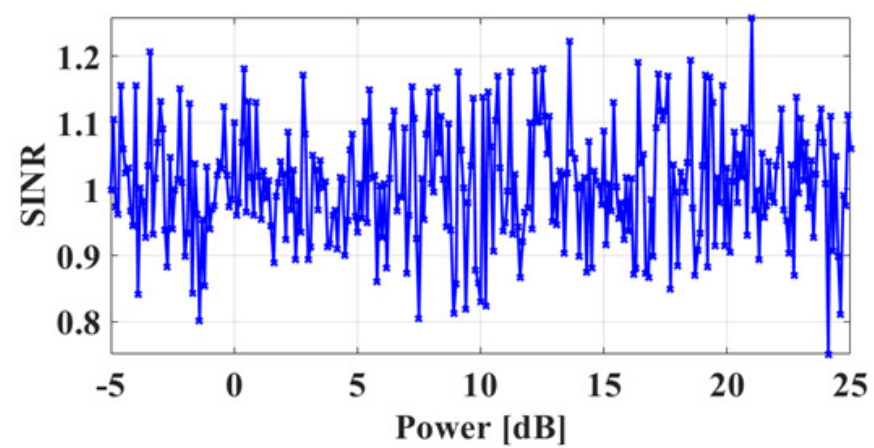

Fig. 6. Limited relaying assistance from non-cognitive user to cognitive transmission with assistance

\section{A Maximum Cognitive Cooperation Case}

In this case, the primary user's transmission is assisted by cognitive users. In Figs. 4 and 5, the SINR SINR vs. Power [dB] graph is shown. It can be seen in maximum cognitive cooperation case (Fig. 4) even at low power, a 1.4 increase in SINR is observed as compared to the no assistance (Fig. 5). This SINR increase of PU results because of relaying of cognitive users because more cognitive users contributed to increase in SINR of non-cognitive user. Another factor is because the power of interference cognitive users is also low, hence, higher SINR is achieved. The case of no or very weak cognitive relay under low interference is discussed in the next section. 


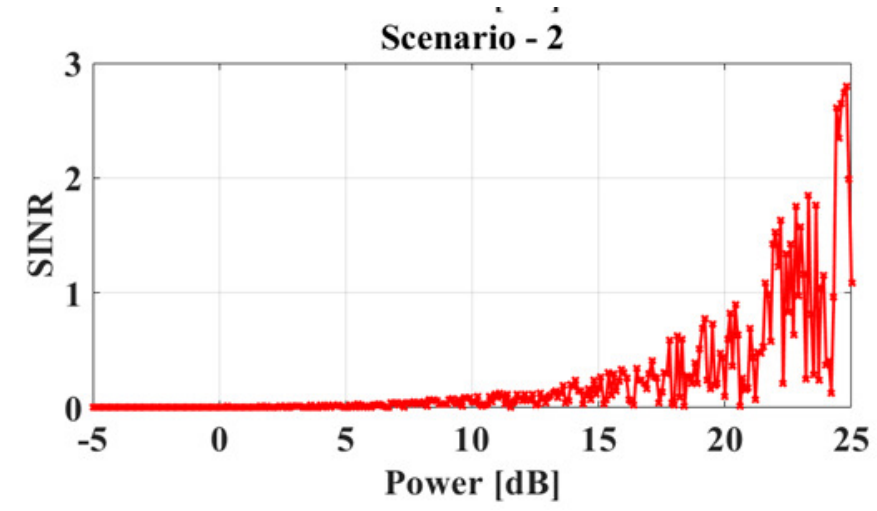

Fig. 7. Limited relaying assistance from non-cognitive user to cognitive transmission with no assistance.

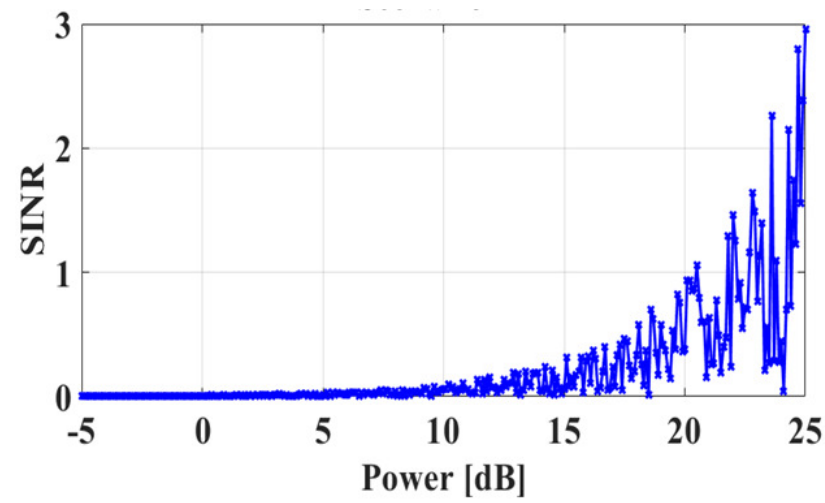

Fig. 8. Interfering users overpowers non-cognitive user transmissions with assistance

\section{B Limited Cognitive Cooperation Case}

A case of limited relaying assistance from non-cognitive user to cognitive transmission is shown in the Figs. 6 and 7. Due to cognitive user's limited assistance through relaying, there is only marginal increase in SINR of PU. It can be observed that because cognitive users' contribution is minimal, the increase in SINR of non-cognitive user is low as compared to the maximum cognitive cooperation scenario. Even, in this case, the power of interference cognitive is comparable to the maximum cognitive cooperation scenario. The impact of increase of the cognitive interference on the primary user is presented in the next section. 


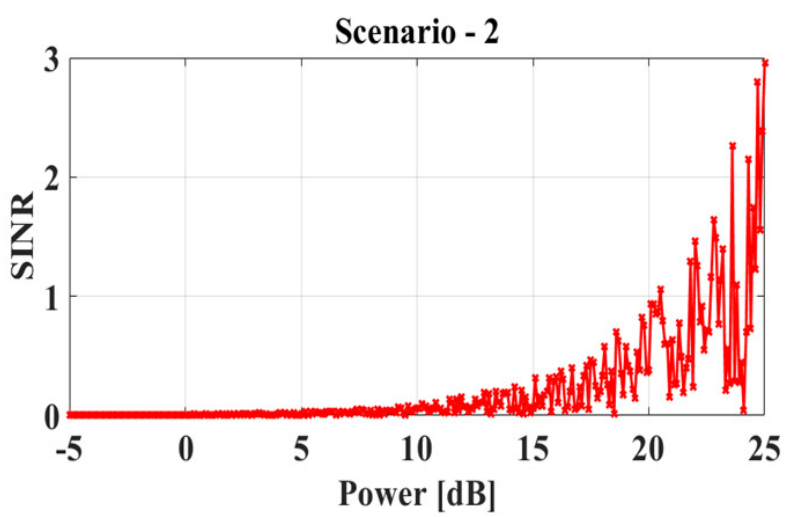

Fig. 9. Interfering users overpowering non-cognitive user transmissions with no-assistance

\section{C Cognitive Interference Case}

In this case, the power of interfering users is increased such that that the sever impacts are observed. The case of interfering users overpowering the non-cognitive user transmissions is shown in Figs 8 and 9. It can be observed that it eliminated the positive effects of relaying, resulting in poor system performance. Here, the CDSSS power control mechanism (Section 4) can be employed as a solution to keep the power of cognitive users under a thresh hold in order to ensure that the operation of PU can continue unhampered.

\section{Future Work}

The successive multiuser detection CDSSS, cognitive relaying, and cooperation among cognitive users make it a candidate transmission technology for cognitive radio systems in digital agriculture applications. It can effectively decode multiple PUs with successive multiuser detection technique which leads to effective spectrum utilization. In CDSSS, the cognitive users adapt to different transmission environments with the help of its effective power and rate control algorithm that has been developed by keeping in view the outage and power constraints of a PU. Many IEEE standards use direct sequence spread spectrum as their physical layer. Therefore, the CDSSS can easily inter-operate with existing systems as compared to other technologies. In CDSSS, the support for multiuser access and immunity from narrow band interference is already inherent in the system design.

One major challenge to CDSSS is synchronization. The success of CDSSS depends greatly on the fact that cognitive user achieves fine synchronization with non-cognitive user for accurate decoding. Synchronization errors can jeop- 


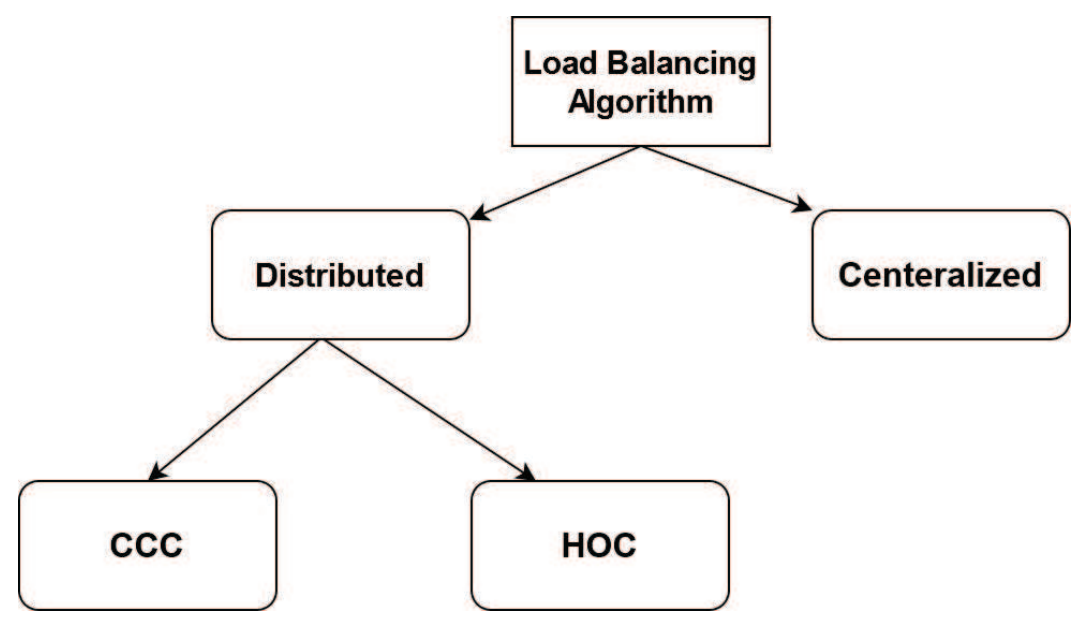

Fig. 10. Classification of load balancing algorithms.

ardize the reliability of the whole system. Cooperation among cognitive users is very important. Therefore, the correct information exchange among cognitive users is also crucial to success. Another challenge to CDSSS is non-cognitive user emulation attack. In this attack, another cognitive user can emulate the characteristics of non-cognitive user and consume resources. In [5], a transmitter verification scheme has been proposed which provides defense against primary user emulation attack in cognitive radio network. A non-cognitive user may be hidden due to multipath fading and shadowing, which leads to difficulties in detection, decoding and relaying and consequently cognitive users have only incomplete information about presence of PU in the network.

\section{Load Balancing and Optimization in the Large and Diverse Agricultural Field}

In this section, a load balancing and optimization approach is presented to enhance the performance of a cognitive radio performance in digital agriculture. This technique is useful to achieve two objectives: 1) load balancing between PUs, and cognitive users (SU), and 2) relaying messages for PU using SU as a relay agent.

\section{A Related Work}

As shown in Fig. 10, load balancing algorithms can be divided into two major categories: the Distributed Load Balancing (Distributed Load Balancing (DLB) ) and Centralized Load Balancing Algorithms (Centralized Load Balancing Algorithms (CLB)). As discussed in Section 1, this method divides an agricultural field into multiple cluster with each cluster having Base station (Base Station (BS)) of its own. The DLBs runs inside the base station (BS). 
In DLB, BSs share information with each other by establishing a direct BS - BS connection to perform either BS-BS user handover (HO) or by allowing user to connect to different BS using SUs as a relay node. In CLB, these tasks are performed by core network, out of the Radio Access Network (Radio Access Network (RAN)). This paper uses DLB class of load balancing for digital agriculture applications.

Traditionally, work done in the DLB predicts the overloaded cell and dynamically adjust parameters to perform $\mathrm{HO}$ and achieve equilibrium between BSs. DLB can further be classified into: Cell Coverage Control (Cell Coverage Control (CCC)) and Hand-Over Control (Hand-Over Control (HOC)) (Fig. 10). In CCC, coverage area of cell dynamically shrinks (to reduce load) or expands (to reach more users) when the cell is under loaded or overloaded, respectively. This helps adjust the cell size based on the sensor density and farm equipment and machinery in the agricultural fields. In [49], authors proposed a technique which constantly monitors the load of its attached resources, i.e., users. It the BS gets overloaded, it optimizes the transmission power of the overloaded BS to decrease its load and gets help from the neighboring clusters. In [33], authors uses a fairness metric Jain's Fairness Index [23] to ensure the fairness for load between the BSs.

The HOC uses thresholds to dynamically perform HO between users and BS. HO is performed based on multiple functional parameters (e.g., state of the network and type of transmitted data). The proposed approach falls under HOC class because of its ability to control link of individual user without inflicting any changes on the performance of other users. In [28], author minimize the overhead incurred by $\mathrm{HO}$ of users by assigning weights to load balancing and signaling overhead. Another approach is to formulate the load balancing problem as an integer programming problem which optimize the load distribution among BSs [16]. [45] formulates load balancing as a Integer Linear Programming problem and uses the Jain's Fairness Index as a metric for performance measure. [48] uses game theory in to formulate load balancing which involves assigning a price factor to each BS and try to reach a state where each BS shares equal traffic load. The already proposed works uses single hop connectivity for HOs with an assumption of direct connections to BS. To the best of our knowledge, this work is different from other currently proposed work in that it considers multihop load balancing with SU relaying the information from overloaded cell while increasing the fair resource utilization and average throughput of the network.

\section{Methodology}

The proposed approach falls under the category of Hand-Over Control (HOC) load balancing mechanism. It differs from others in that it considers multi-hop connectivity while performing HOs between BSs. It considers two-hop connectivity for relaying instead of single hop for HO between PUs and BSs (from underground sensor to aboveground radio to barn based data aggregator). The 

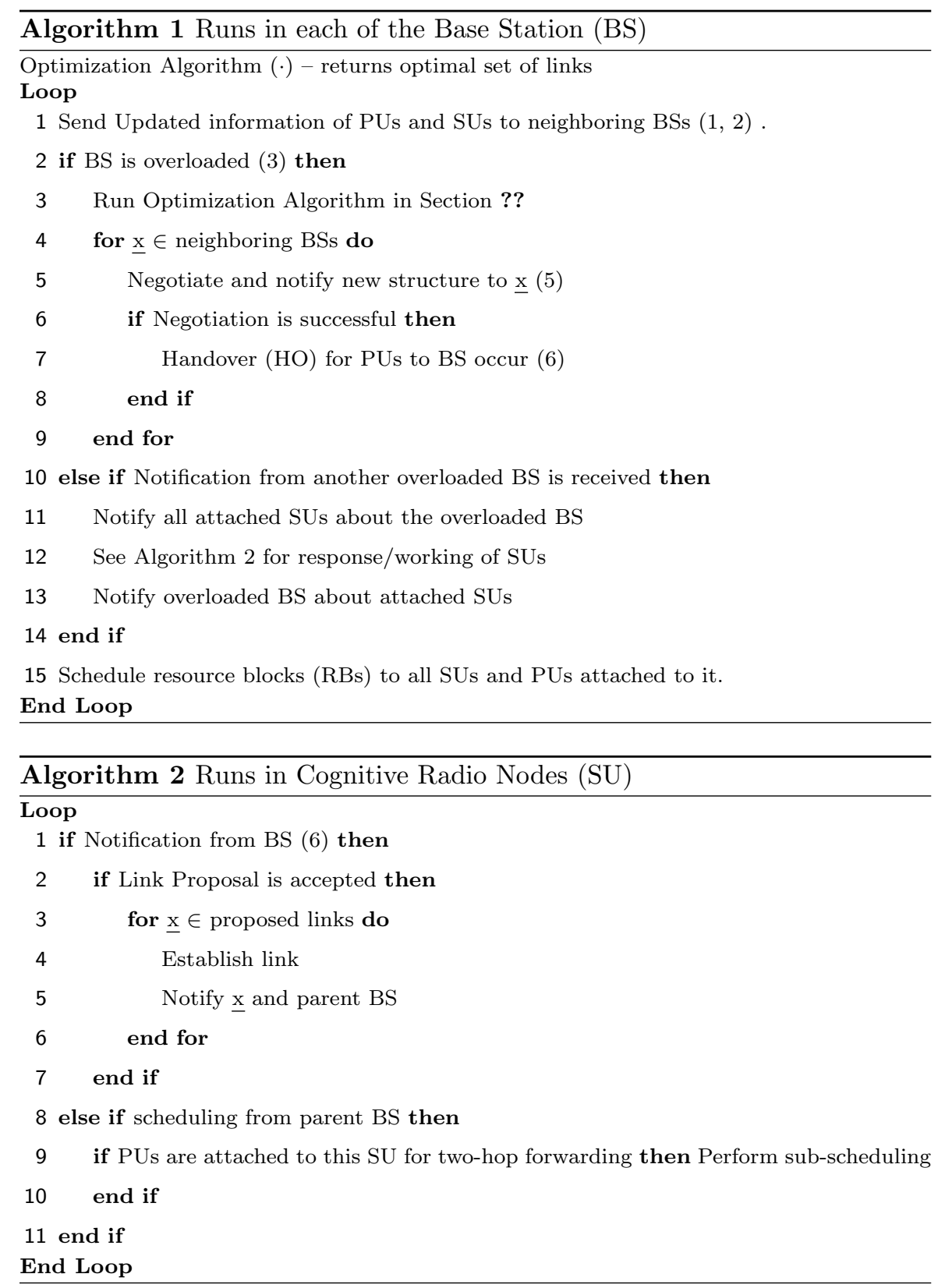


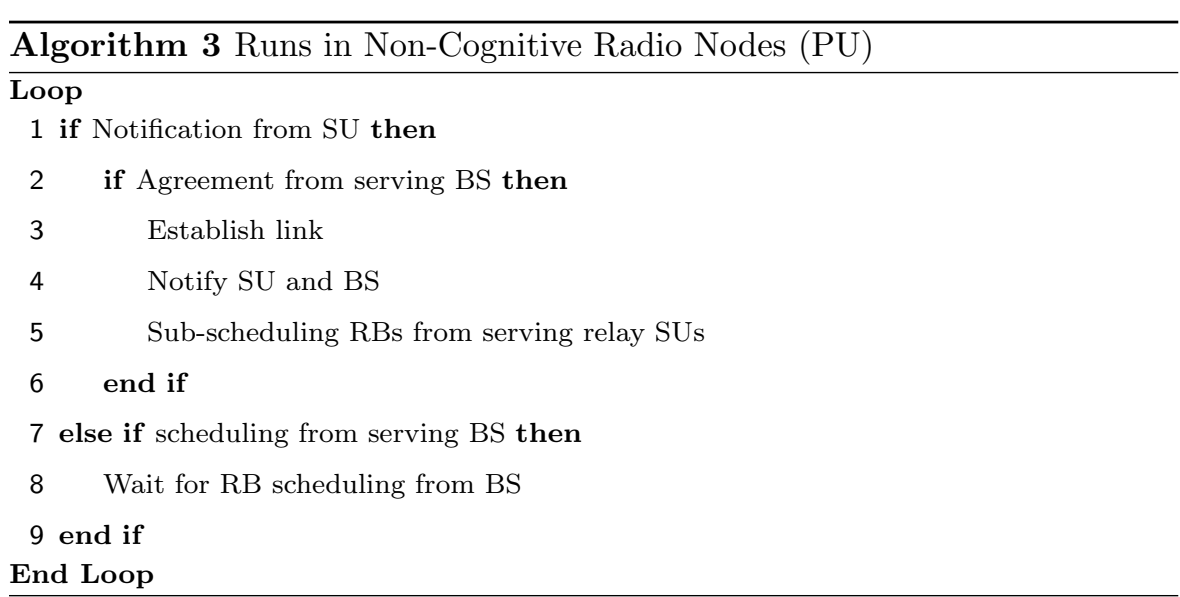

proposed technique detects an overloaded base station and try to reduce its load by diverting the user traffic to neighboring under loaded BS. This is useful to accommodate different type of terrains in farms. The major components of this technique are: 1) Non-Cognitive User (PUs), 2) Base Stations (BS) and 3) Cognitive Users (SU). SUs also act as relay node to provide connectivity between PUs and BSs. The detailed design is explained in the next section where the two important blocks of the procedures are discussed. The Algorithm 1, 2, and 3 shows the complete functionality of the proposed technique with respect to BS, SU and PU respectively. Fig. 12 shows the process of message exchange between the various actors involved in the developed approach. The algorithm starts out with BSs communicating the information about all connected SUs SU and PUs with other BSs in different cluster [see Fig. 12: step (1) and (2)]. The purpose of this step is to have a knowledge of updated traffic status of the cluster. In this case, a BS will be considered as operating normal if it is underloaded. Initially, all BSs are operating normal. When a BS becomes overloaded [see Fig. 12: step (3)] due to excess of traffic, it solves the optimization problem (Fig. 12: step (4)). The purpose of this approach is to find the set of optimum links which are needed to maximize performance cost. After selecting an optimal set of links according to a pre-decided optimization criteria, the overloaded BS negotiates this set of links with other BSs [see Fig. 12: step (5)]. If a conflict arise because of the other BS also being overloaded, BS with the highest workload will have authority to impose its calculated set of links. After agreement between overloaded and it neighboring BSs, each neighbor BS choose a SU [see Fig. 12: step (6)] (acting as an ad-hoc relay node) in their corresponding cluster. After selecting SU, the selected SU invites PUs in overloaded cells to connect with them. Finally, PUs perform HO after notifying the overloaded cell. This approach is useful to reduce load in higher sensor density areas. After link establishment, BS will schedule the set of resource blocks (Resource Block (RB)) to its attached users (PUs and SUs). RBs here represents a smallest unit of resource that can be allocated. SUs, acting as a 
Base Station

relay agent/node, will further sub-schedule its assigned RBs to attached PUs from overloaded cluster. Different scheduling methods can be used, however, this proposal is limited to Round-Robin scheduling. Optimization of scheduler performance or proposing new scheduling mechanism can be considered as a future problem.

Accordingly, BS assigns RBs to its directly attached PUs and SUs (a relay node local to each cluster). SUs may also have some (zero or more) PUs attached to it. SUs relays the information from parent BS to these attached PUs. This process of re-scheduling of subset of RBs by SUs is known as subscheduling. Fig. 11 shows this phase of the algorithm. The round robin approach is used by SUs as a scheduling algorithm during sub-scheduling phase.

\section{Conclusions}

The paper presented two different approaches of improving cognitive radios implementation in agricultural setup. The CDSSS approach works by acquiring blind synchronization, successive multiuser decoding, relaying, and cooperation by information exchange among PU. It holds promise for efficient spectrum utilization and solution to spectrum scarcity problem in the field of 


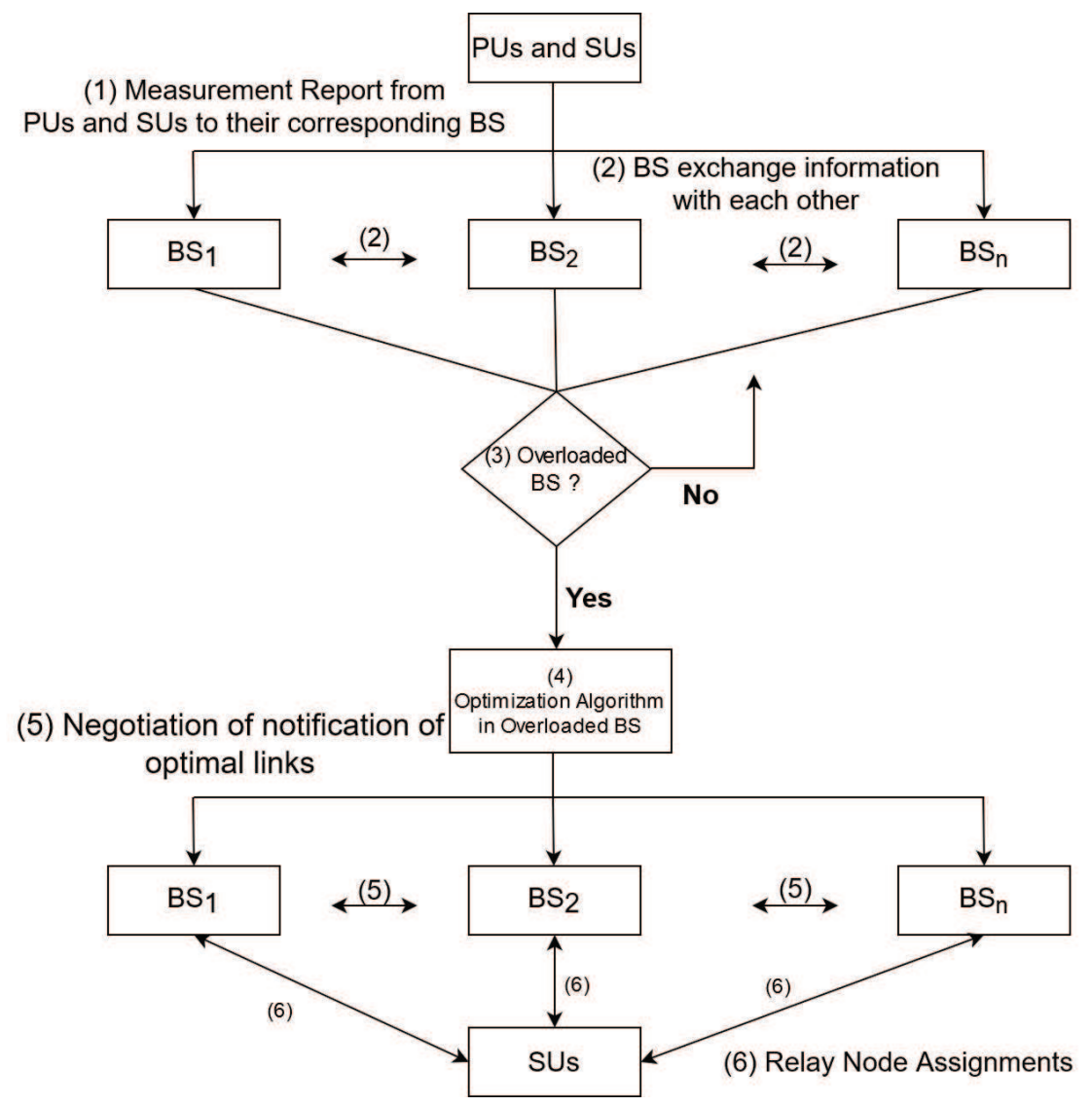

Fig. 12. The message exchange flow between different components of the network

digital agriculture. The CDSSS also realizes the cognitive novel radio concept and introduce new capabilities to effectively utilize the white spaces in agricultural farms. More emphasis should be given to solve challenges to CDSSS implementation.

A load balancing and optimization approach is presented to enhance cognitive radio performance in digital agriculture. The purpose of the technique is to increase the throughput of the network by implementing load balancing. It does so relaying messages for PU in overloaded cluster through a SU operating as a relay agent to underloaded cluster. It can be very useful in farm settings where a relay $\mathrm{SU}$ can be attached to a moving farm equipment to transfer data from one overloaded cluster to another underloaded cluster. 


\section{Declaration}

This article is an extended version of our conference paper: A Cooperative Overlay Approach at the Physical Layer of Cognitive Radio for Digital Agriculture in Proc. 3rd International Balkan Conference on Communications and Networking (2019 BalkanCom), Skopje, North Macedonia, June 2019 [39]. Available at: https://docs.lib.purdue.edu/cit_articles/16/.

The contribution of the initial conference paper was to improve the physical layer architecture of cognitive radios so that it can be efficiently utilized for reusing the scarce frequency spectrum resource. This work significantly extend the original paper by improving the quality of Service (Quality of Service (QoS)) by performing load balancing, through relaying, between the cognitive and PU in the spectrum. The major contribution of the paper is to improve the operation of cognitive radios in digital agriculture. Furthermore, we declare that, currently, this article is not submitted to any other journal.

\section{A Availability of data and material}

All data have been presented in the paper in form of graphs.

\section{B Competing interests}

No competing interests.

\section{C Funding}

This work did not receive any external funding.

\section{D Authors' contributions}

U.R. contributed to optimization problem. A.S. contributed to performance evaluations. U.K. contributed to system design.

\section{References}

1. 802.22, I.: Working group on wireless regional area networks. URL http://www. ieee802. org/22/

2. Andrews, S., Gerdes, R.M., Li, M.: Towards physical layer identification of cognitive radio devices. In: 2017 IEEE Conference on Communications and Network Security (CNS), pp. 1-9. IEEE (2017)

3. Cabric, D., Brodersen, R.W.: Physical layer design issues unique to cognitive radio systems. In: 2005 IEEE 16th International Symposium on Personal, Indoor and Mobile Radio Communications, vol. 2, pp. 759-763. IEEE (2005) 
4. Chahrour, H., Rajan, S., Dansereau, R., Balaji, B.: Hybrid spread spectrum orthogonal waveforms for mimo radar. In: 2018 IEEE Radar Conference (RadarConf18), pp. 10101014. IEEE (2018)

5. Chen, R., Park, J.M., Reed, J.H.: Defense against primary user emulation attacks in cognitive radio networks. IEEE Journal on selected areas in communications 26(1), 25-37 (2008)

6. Chen, Y.W., Peng, P.C., Huang, L., Chang, G.K.: Reliable multi-user uplinks in fiberwireless integrated network using quasi-orthogonal chirp spreading ofdm. In: 2019 IEEE MTT-S International Microwave Conference on Hardware and Systems for 5G and Beyond (IMC-5G), pp. 1-3. IEEE (2019)

7. Chronopoulos, A.T., Musku, M.R., Penmatsa, S., Popescu, D.C.: Spectrum load balancing for medium access in cognitive radio systems. IEEE Communications Letters 12(5), 353-355 (2008)

8. Commission, F.C., et al.: Second memorandum opinion and order. FCC 10-174 (2010)

9. Connor, R.: The United Nations world water development report 2015: water for a sustainable world, vol. 1. UNESCO publishing (2015)

10. Dalvi, A., Swamy, P., Meshram, B.: Challenges of spectrum sensing techniques for cognitive radio. In: Proceedings of the International Conference \& Workshop on Emerging Trends in Technology, pp. 972-973 (2011)

11. Devroye, N., Mitran, P., Tarokh, V.: Achievable rates in cognitive radio channels. IEEE Transactions on information theory 52(5), 1813-1827 (2006)

12. Digham, F.F.: Joint power and channel allocation for cognitive radios. In: 2008 IEEE Wireless Communications and Networking Conference, pp. 882-887. IEEE (2008)

13. Fang, Z.: New detection methods for transmission at faster-than-nyquist rates using quasi-orthogonal sequences (2018)

14. FCC, E.: Docket no. 08-260,". Second Report and Order and Memorandum Opinion and Order," Nov (2008)

15. Ghavami, S., Alikhanian, H., Abolhassani, B., Rad, H.S.: Blind multiuser data estimation in asynchronous and unequal power ds-ss systems without any prior knowledge of spreading sequences. In: 2009 IEEE Sarnoff Symposium, pp. 1-6. IEEE (2009)

16. Giovanidis, A., Liao, Q., Stańczaky, S.: A distributed interference-aware load balancing algorithm for lte multi-cell networks. In: 2012 International ITG Workshop on Smart Antennas (WSA), pp. 28-35. IEEE (2012)

17. Goldsmith, A., Jafar, S.A., Maric, I., Srinivasa, S.: Breaking spectrum gridlock with cognitive radios: An information theoretic perspective. Proceedings of the IEEE 97(5), 894-914 (2009)

18. Gong, X., Yuan, W., Liu, W., Cheng, W., Wang, S.: A cooperative relay scheme for secondary communication in cognitive radio networks. In: IEEE GLOBECOM 20082008 IEEE Global Telecommunications Conference, pp. 1-6. IEEE (2008)

19. Hassan, F., Ajmal, S., Khan, Z., Hassan, U.: Analysis on multiple access capability of hybrid spread spectrum system with optimal sequences-a review of performance parameters trade-off. Analysis on Multiple Access Capability of Hybrid Spread Spectrum System with Optimal Sequences-A Review of Performance Parameters Trade-off 12(1), 9-9 (2018)

20. Haykin, S.: Cognitive radio: brain-empowered wireless communications. IEEE journal on selected areas in communications 23(2), 201-220 (2005)

21. Hoang, A.T., Liang, Y.C.: A two-phase channel and power allocation scheme for cognitive radio networks. In: 2006 IEEE 17th International Symposium on Personal, Indoor and Mobile Radio Communications, pp. 1-5. IEEE (2006)

22. Honig, M., Madhow, U., Verdu, S.: Blind adaptive multiuser detection. IEEE Transactions on Information Theory 41(4), 944-960 (1995)

23. Jain, R.K., Chiu, D.M.W., Hawe, W.R., et al.: A quantitative measure of fairness and discrimination. Eastern Research Laboratory, Digital Equipment Corporation, Hudson, MA (1984)

24. Jayapalan, A., Savarinathan, P., Abarna, L., Abinaya, K.: Role of cognitive radio to improve agriculture. In: 2019 International Conference on Vision Towards Emerging Trends in Communication and Networking (ViTECoN), pp. 1-6. IEEE (2019) 
25. Kang, X., Zhang, R., Liang, Y.C., Garg, H.K.: Optimal power allocation strategies for fading cognitive radio channels with primary user outage constraint. IEEE Journal on Selected Areas in Communications 29(2), 374-383 (2011)

26. Kim, Y., de Veciana, G.: Joint network capacity region for cognitive networks heterogeneous environments and rf-environment awareness. IEEE Journal on Selected Areas in Communications 29(2), 407-420 (2011)

27. Kuroyanagi, N., Suehiro, N., Ozawa, S., Tomita, M., Otake, K., Takahashi, M.: Cdma communications system using multiple spreading sequences (2007). US Patent 7,245,650

28. Li, Z., Wang, H., Pan, Z., Liu, N., You, X.: Joint optimization on load balancing and network load in 3gpp lte multi-cell networks. In: 2011 International Conference on Wireless Communications and Signal Processing (WCSP), pp. 1-5. IEEE (2011)

29. Lupas, R., Verdu, S.: Near-far resistance of multiuser detectors in asynchronous channels. IEEE transactions on Communications 38(4), 496-508 (1990)

30. Maric, I., Yates, R.D., Kramer, G.: Capacity of interference channels with partial transmitter cooperation. IEEE Transactions on Information Theory 53(10), 3536-3548 (2007)

31. Mitola, J.: Cognitive radio. an integrated agent architecture for software defined radio. (2002)

32. Niyato, D., Hossain, E.: A game-theoretic approach to competitive spectrum sharing in cognitive radio networks. In: 2007 IEEE Wireless Communications and Networking Conference, pp. 16-20. IEEE (2007)

33. Ronoh, K., Mengistie, A.: Load balancing in heterogeneous lte-a networks (2012)

34. Sahai, A., Tandra, R., Mishra, S.M., Hoven, N.: Fundamental design tradeoffs in cognitive radio systems. In: Proceedings of the first international workshop on Technology and policy for accessing spectrum, pp. 2-es (2006)

35. Salam, A.: Design of subsurface phased array antennas for digital agriculture applications. In: Proc. 2019 IEEE International Symposium on Phased Array Systems and Technology (IEEE Array 2019). Waltham, MA, USA (2019)

36. Salam, A.: An underground radio wave propagation prediction model for digital agriculture. Information 10(4) (2019). DOI 10.3390/info10040147. URL http://www.mdpi. com/2078-2489/10/4/147

37. Salam, A.: Internet of Things for Sustainable Community Development, 1 edn. Springer Nature (2020). DOI 10.1007/978-3-030-35291-2

38. Salam, A.: Internet of Things in Agricultural Innovation and Security, pp. 71-112. Springer International Publishing, Cham (2020). DOI 10.1007/978-3-030-35291-2\_3. URL https://doi.org/10.1007/978-3-030-35291-2\_3

39. Salam, A., Karabiyik, U.: A cooperative overlay approach at the physical layer of cognitive radio for digital agriculture. In: 3rd International Balkan Conference on Communications and Networking (2019 BalkanCom) (2019)

40. Salam, A., Vuran, M.C., Irmak, S.: Di-sense: In situ real-time permittivity estimation and soil moisture sensing using wireless underground communications. Computer Networks 151, 31-41 (2019)

41. Sandonaris, A., Erkip, E., Aazhang, B.: User cooperation diversity-part ii: Implementation aspects and performance analysis. IEEE Transactions on Communication 51(11), 1939-1948 (2003)

42. Sari, H., Vanhaverbeke, F., Moeneclaey, M.: Increasing the capacity of cdma using hybrid spreading sequences and iterative multistage detection. In: Gateway to 21st Century Communications Village. VTC 1999-Fall. IEEE VTS 50th Vehicular Technology Conference (Cat. No. 99CH36324), vol. 2, pp. 1160-1164. IEEE (1999)

43. Sendonaris, A., Erkip, E., Aazhang, B.: User cooperation diversity. part i. system description. IEEE Transactions on communications 51(11), 1927-1938 (2003)

44. Vuran, M.C., Salam, A., Wong, R., Irmak, S.: Internet of underground things in precision agriculture: Architecture and technology aspects. Ad Hoc Networks 81, 160-173 (2018)

45. Wang, H., Liu, N., Li, Z., Wu, P., Pan, Z., You, X.: A unified algorithm for mobility load balancing in 3gpp lte multi-cell networks. Science China Information Sciences 56(2), $1-11(2013)$

46. Xiang, Z., Yang, W., Pan, G., Cai, Y., Song, Y.: Physical layer security in cognitive radio inspired noma network. IEEE Journal of Selected Topics in Signal Processing 13(3), 700-714 (2019) 
47. Xing, Y., Mathur, C.N., Haleem, M.A., Chandramouli, R., Subbalakshmi, K.: Dynamic spectrum access with qos and interference temperature constraints. IEEE Transactions on mobile computing 6(4), 423-433 (2007)

48. Xu, H., Zhou, X., Chen, Y.: A differential game model of automatic load balancing in lte networks. Wireless personal communications 71(1), 165-180 (2013)

49. Zhang, H., Qiu, X.s., Meng, L.m., Zhang, X.d.: Achieving distributed load balancing in self-organizing lte radio access network with autonomic network management. In: 2010 IEEE Globecom Workshops, pp. 454-459. IEEE (2010)

50. Zhang, J., Jia, J., Zhang, Q., Lo, E.M.: Implementation and evaluation of cooperative communication schemes in software-defined radio testbed. In: 2010 Proceedings IEEE INFOCOM, pp. 1-9. IEEE (2010)

51. Zhang, Q., Jia, J., Zhang, J.: Cooperative relay to improve diversity in cognitive radio networks. IEEE Communications Magazine 47(2), 111-117 (2009)

52. Zhang, Q., Kota, S., Lau, V., Su, W., Kwasinski, A.: Introduction to the issue on cooperative communication and signal processing in cognitive radio systems. IEEE Journal of Selected Topics in Signal Processing 5(1), 1-4 (2010)

53. Zhang, X., Su, H.: Opportunistic spectrum sharing schemes for cdma-based uplink mac in cognitive radio networks. IEEE Journal on Selected Areas in Communications 29(4), 716-730 (2011)

\section{List of Figures}

1 The interaction among cognitive radios and PUs ....... 5

2 The CDSSS system model . . . . . . . . . . . . 6

3 Successive multi-user decoding . . . . . . . . . . . 8

4 SINR vs. Power $[\mathrm{dB}]$ : Non-cognitive user transmission is assisted by cognitive users . . . . . . . . . . . . . . . 11

$5 \quad$ SINR vs. Power $[\mathrm{dB}]$ : Non-cognitive user transmission with noassistance ....................... 12

6 Limited relaying assistance from non-cognitive user to cognitive transmission with assistance . . . . . . . . . . 12

$7 \quad$ Limited relaying assistance from non-cognitive user to cognitive transmission with no assistance. . . . . . . . . . . . 13

8 Interfering users overpowers non-cognitive user transmissions with assistance .................... 13

9 Interfering users overpowering non-cognitive user transmissions with no-assistance . . . . . . . . . . . . . . . . 14

10 Classification of load balancing algorithms. . . . . . . . . . . 15

11 Sub-Scheduling by relay node (SUs) in two hop links . . . . . . 19

12 The message exchange flow between different components of the network ...................... 20 


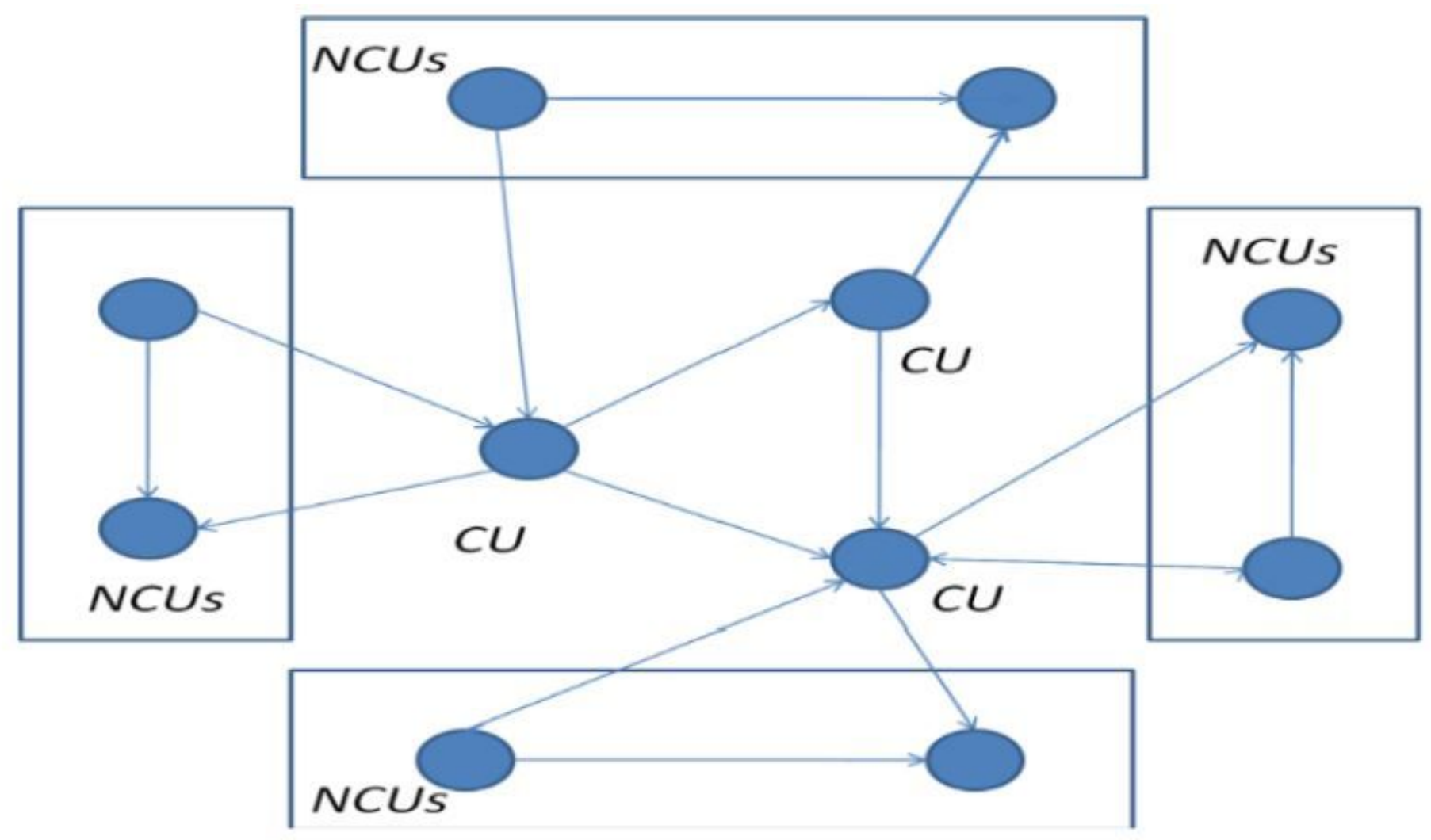

Figure 1

The interaction among cognitive radios and PUs

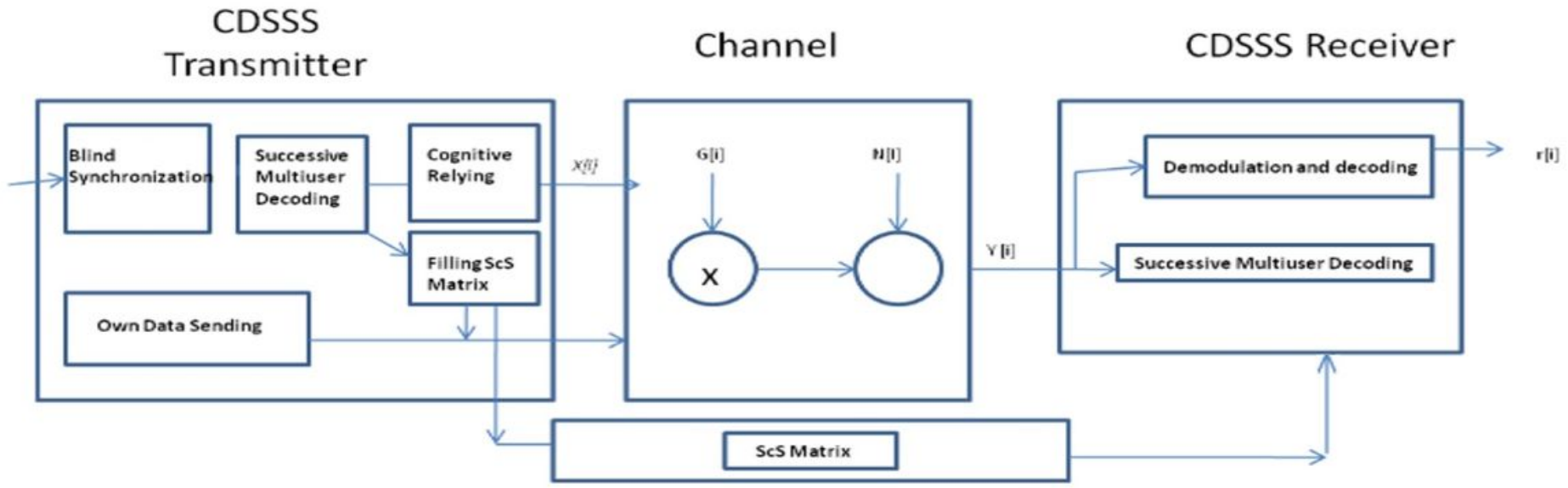

Figure 2

The CDSSS system model 


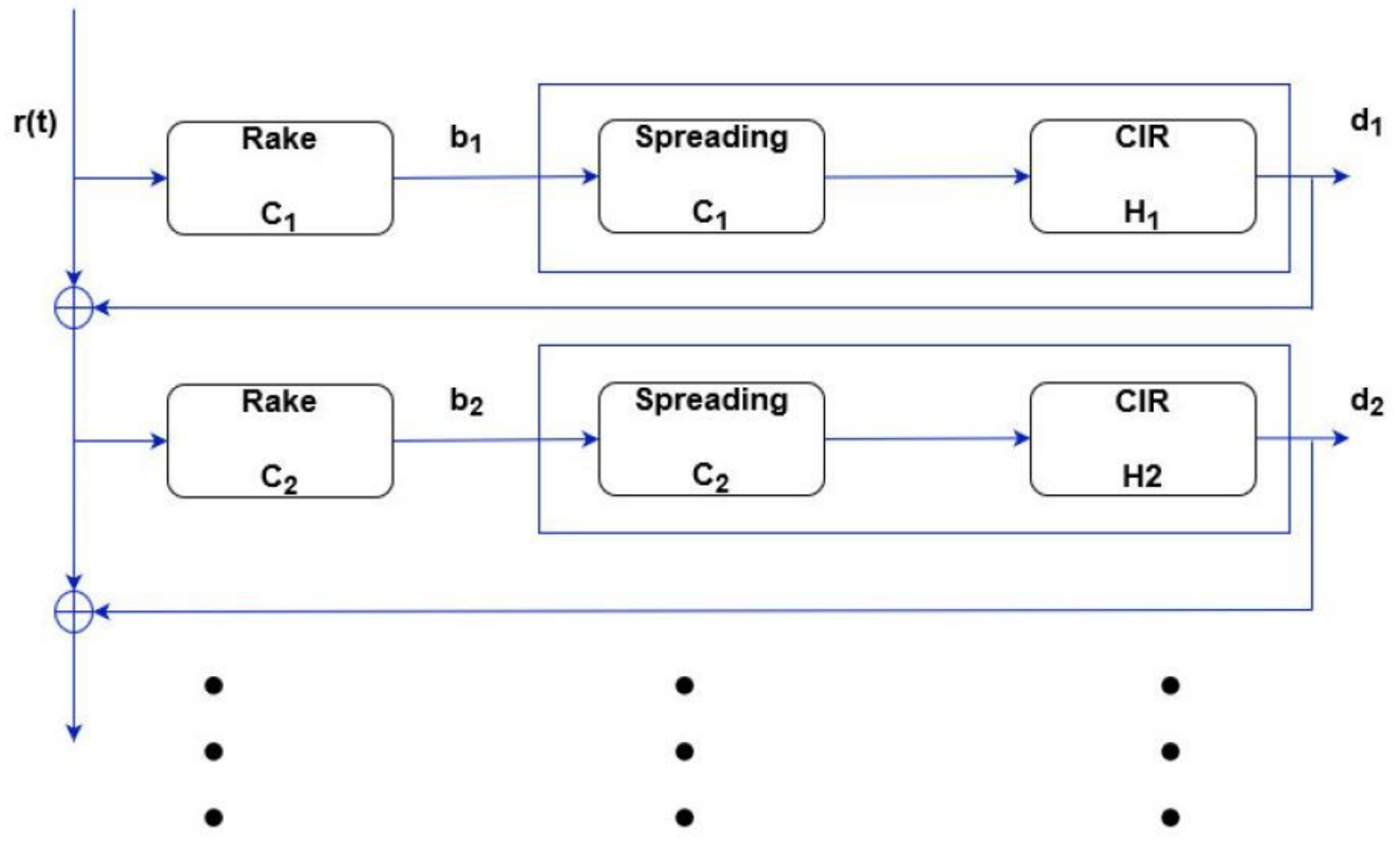

Figure 3

Successive multi-user decoding 


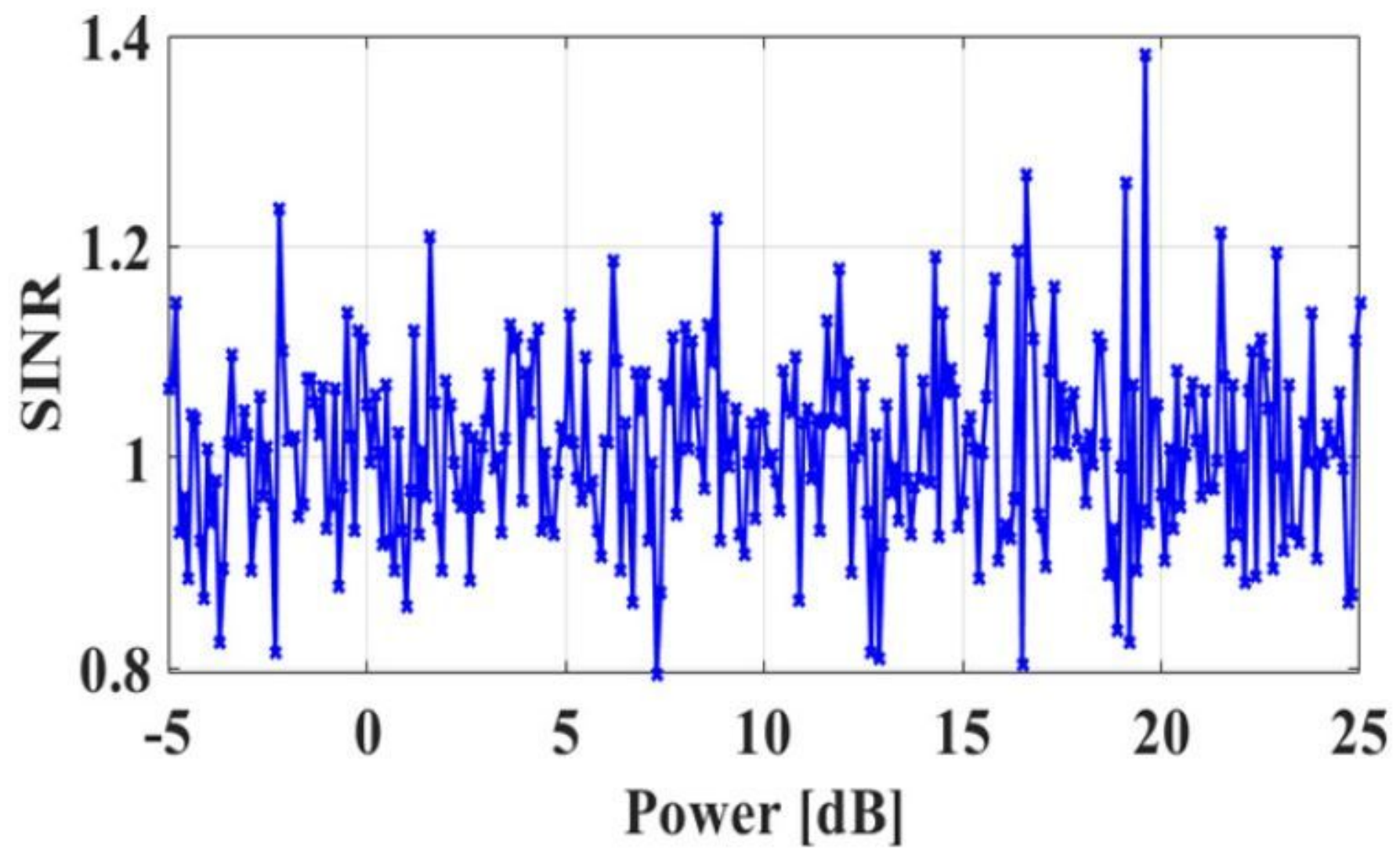

Figure 4

SINR vs. Power [dB] : Non-cognitive user transmission is assisted by cognitive users 


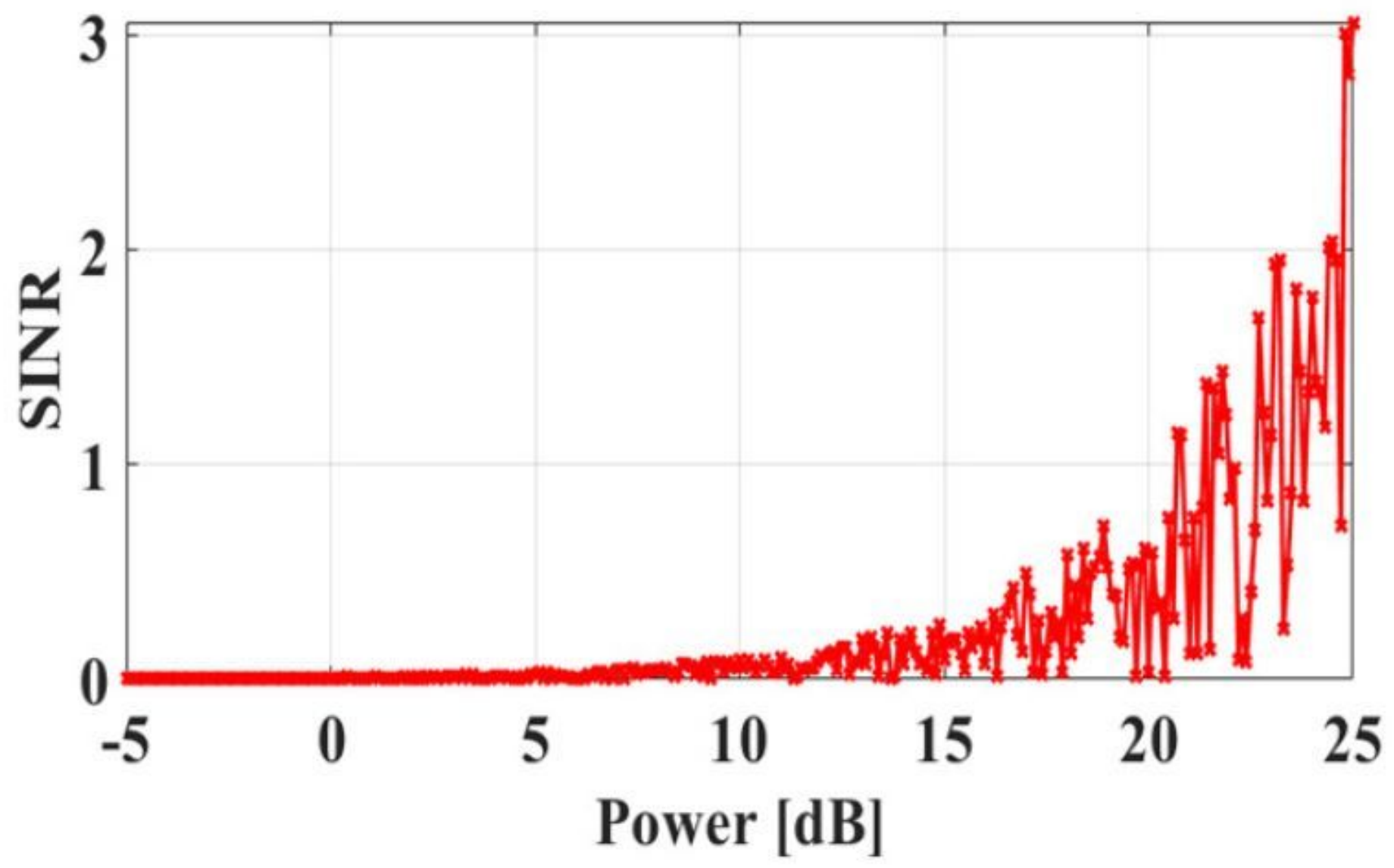

Figure 5

SINR vs. Power [dB]: Non-cognitive user transmission with no-assistance

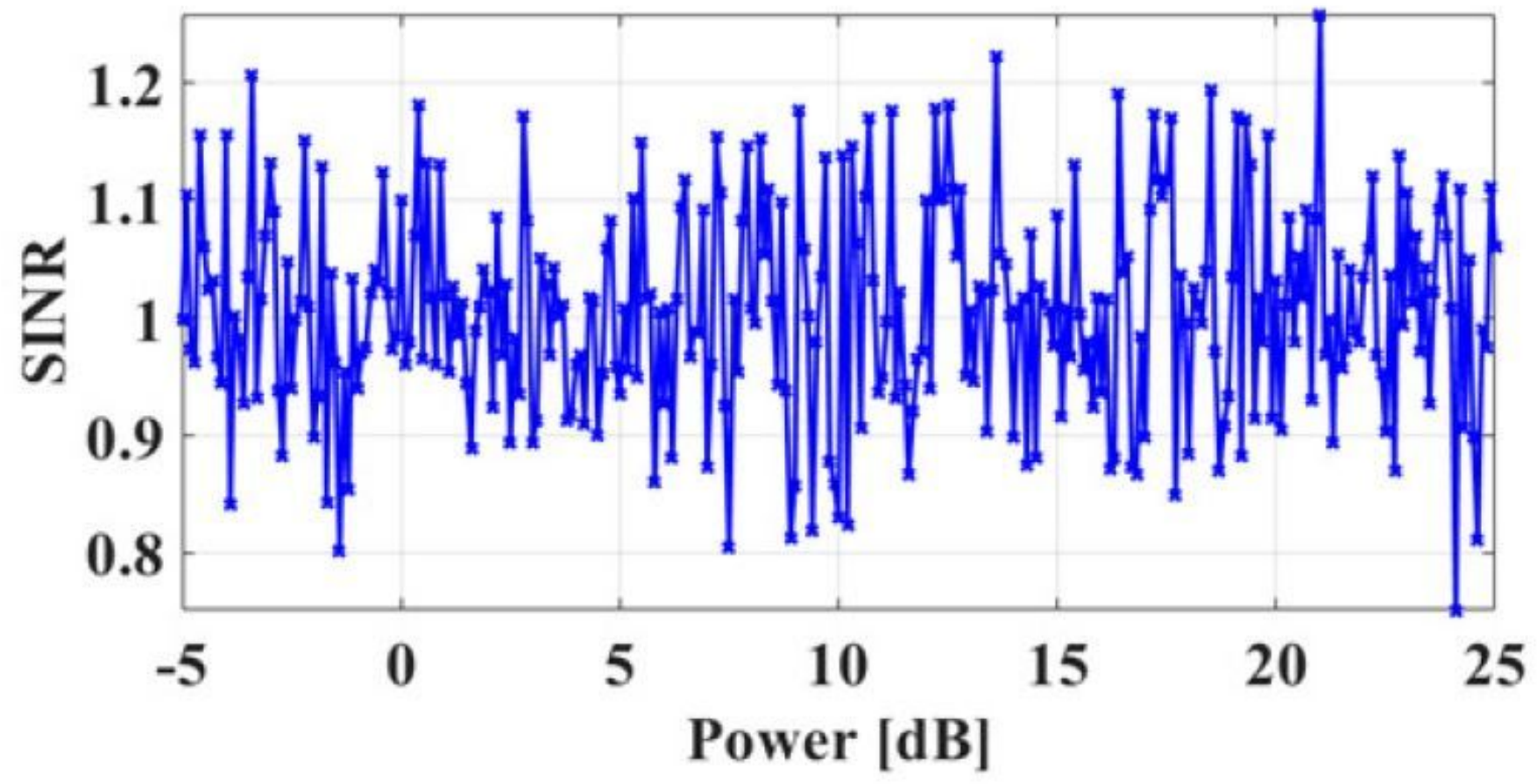


Figure 6

Limited relaying assistance from non-cognitive user to cognitive transmission with assistance

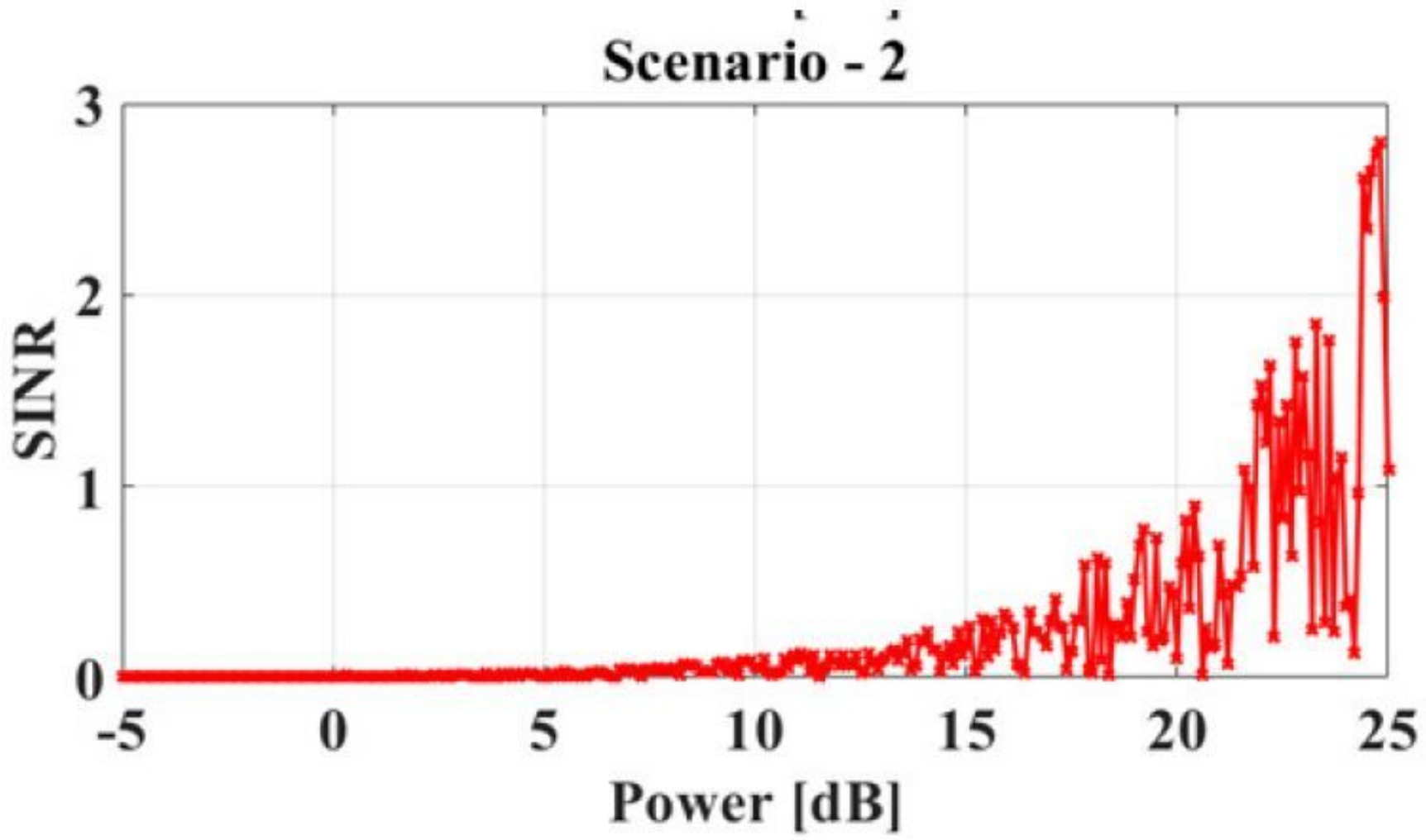

Figure 7

Limited relaying assistance from non-cognitive user to cognitive transmission with no assistance 


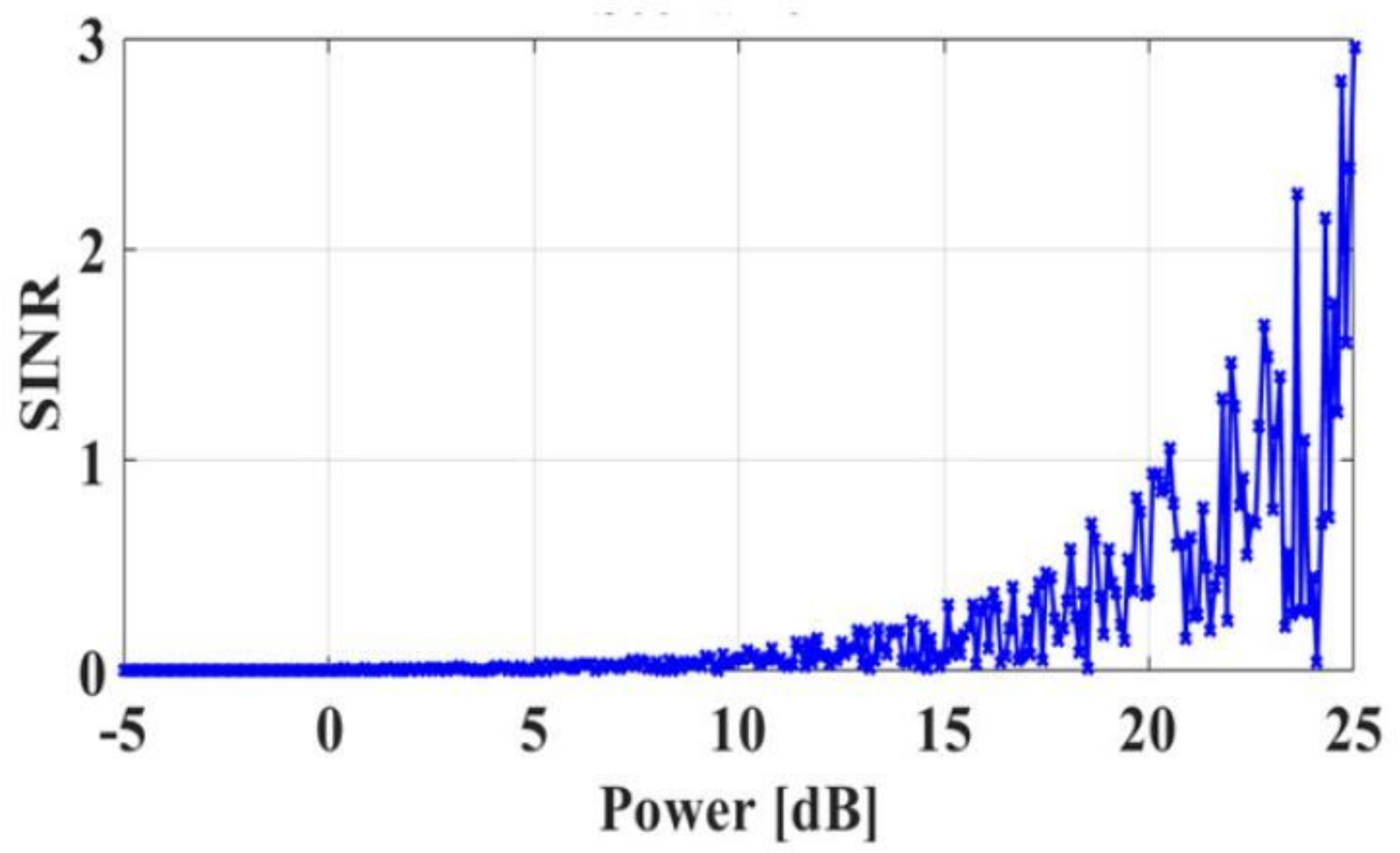

Figure 8

Interfering users overpowers non-cognitive user transmissions with assistance 
Scenario - 2

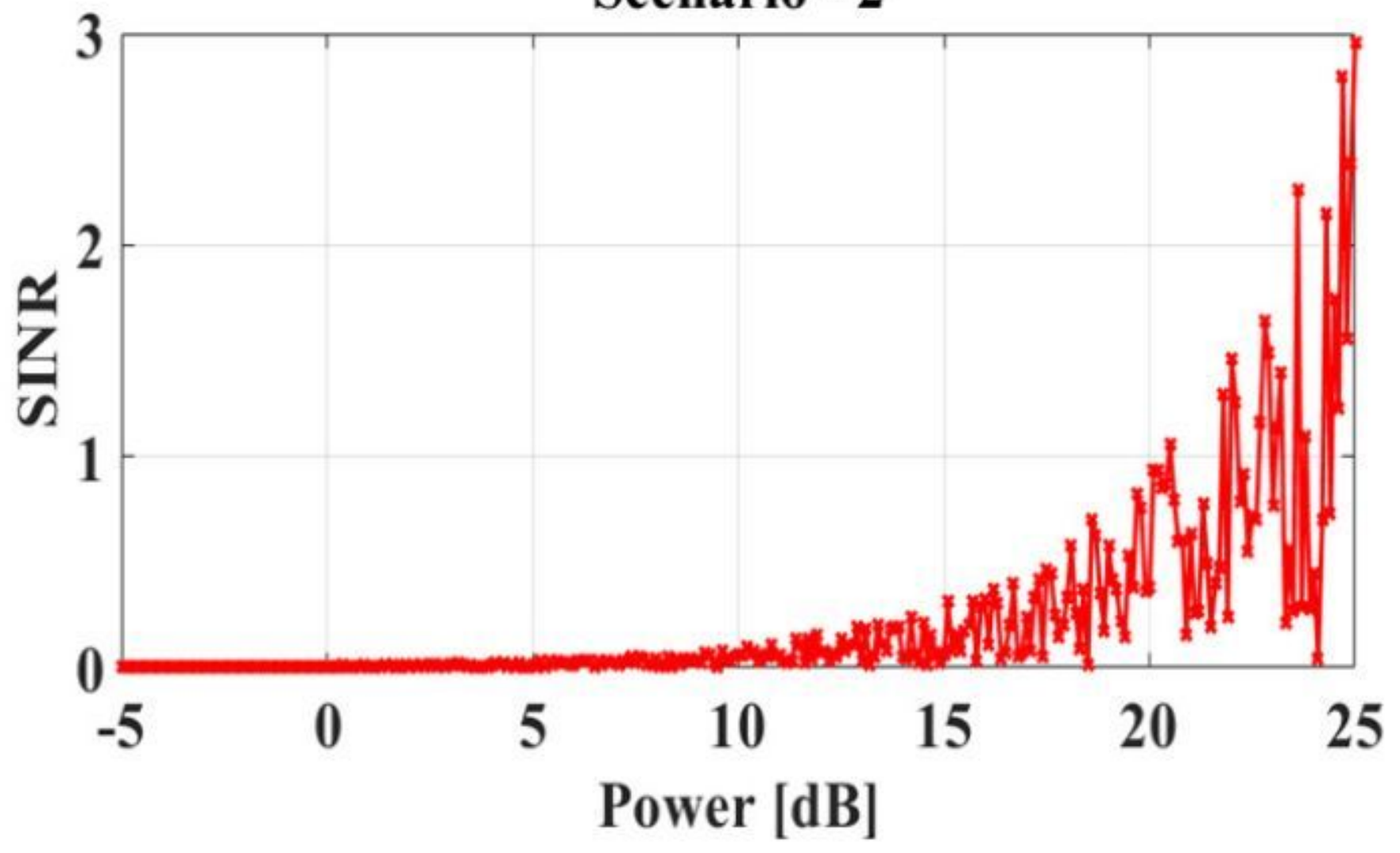

Figure 9

Interfering users overpowering non-cognitive user transmissions with no-assistance

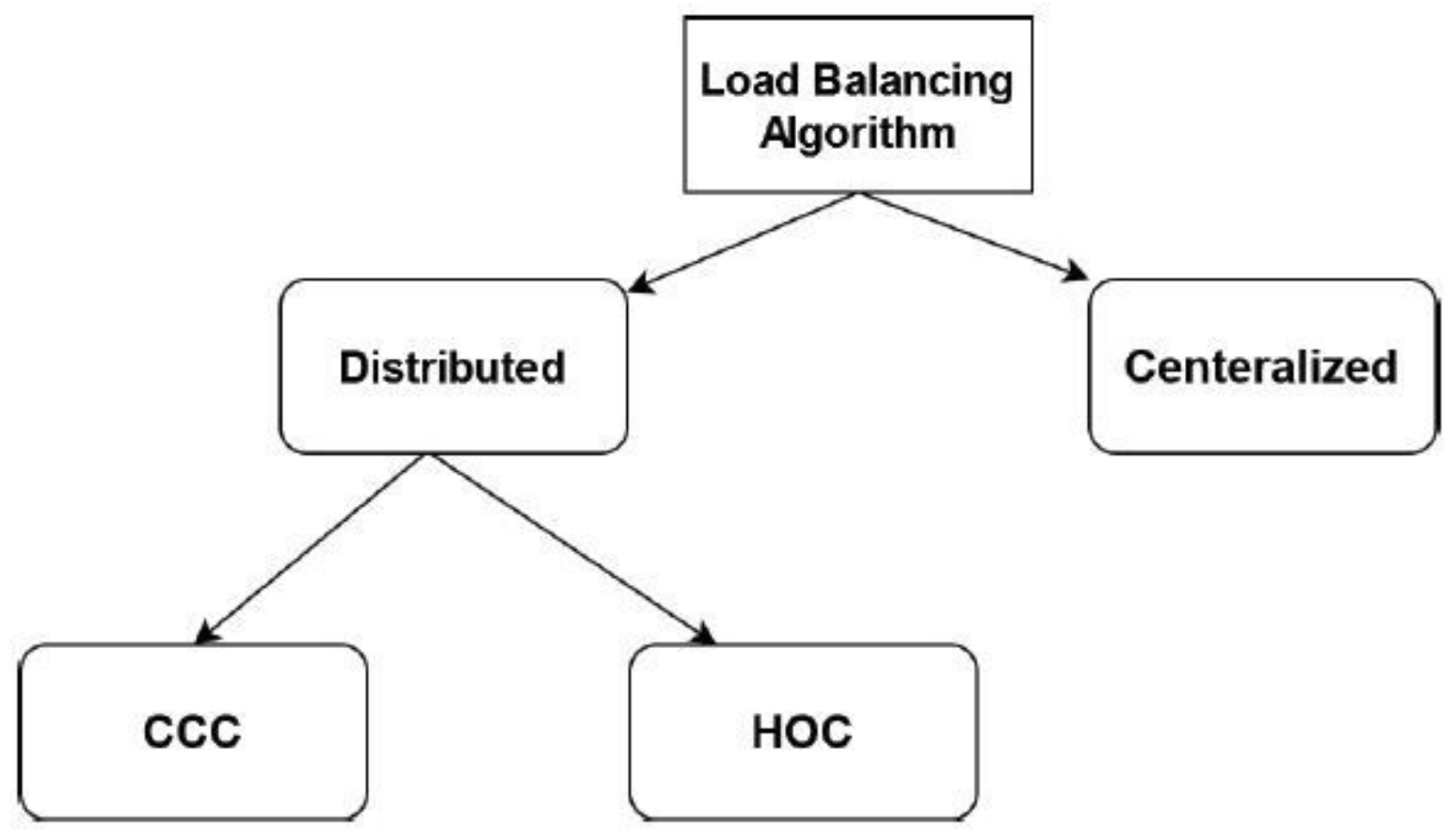

Figure 10 


\section{Base Station}
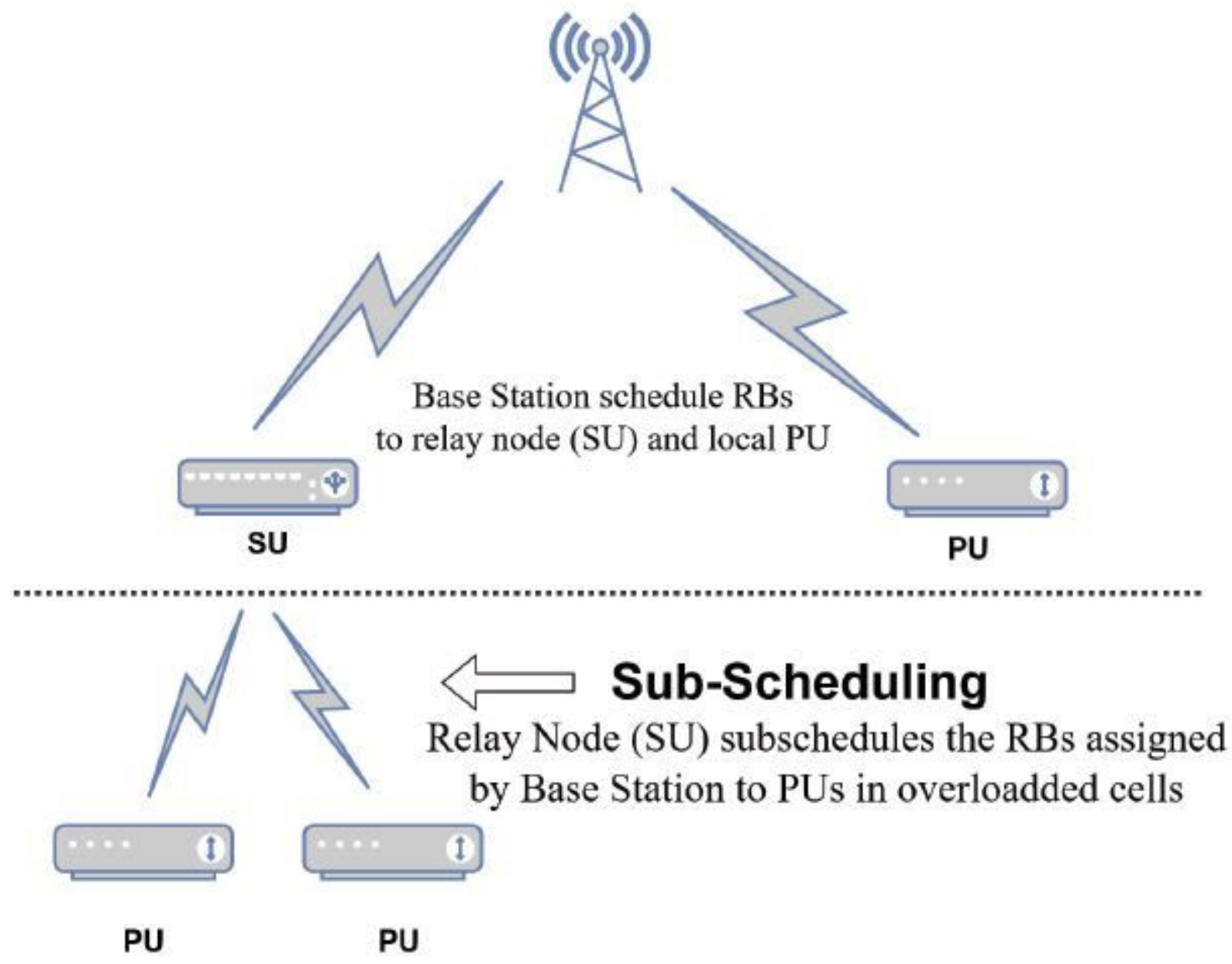

Figure 11

Sub-Scheduling by relay node (SUs) in two hop links 


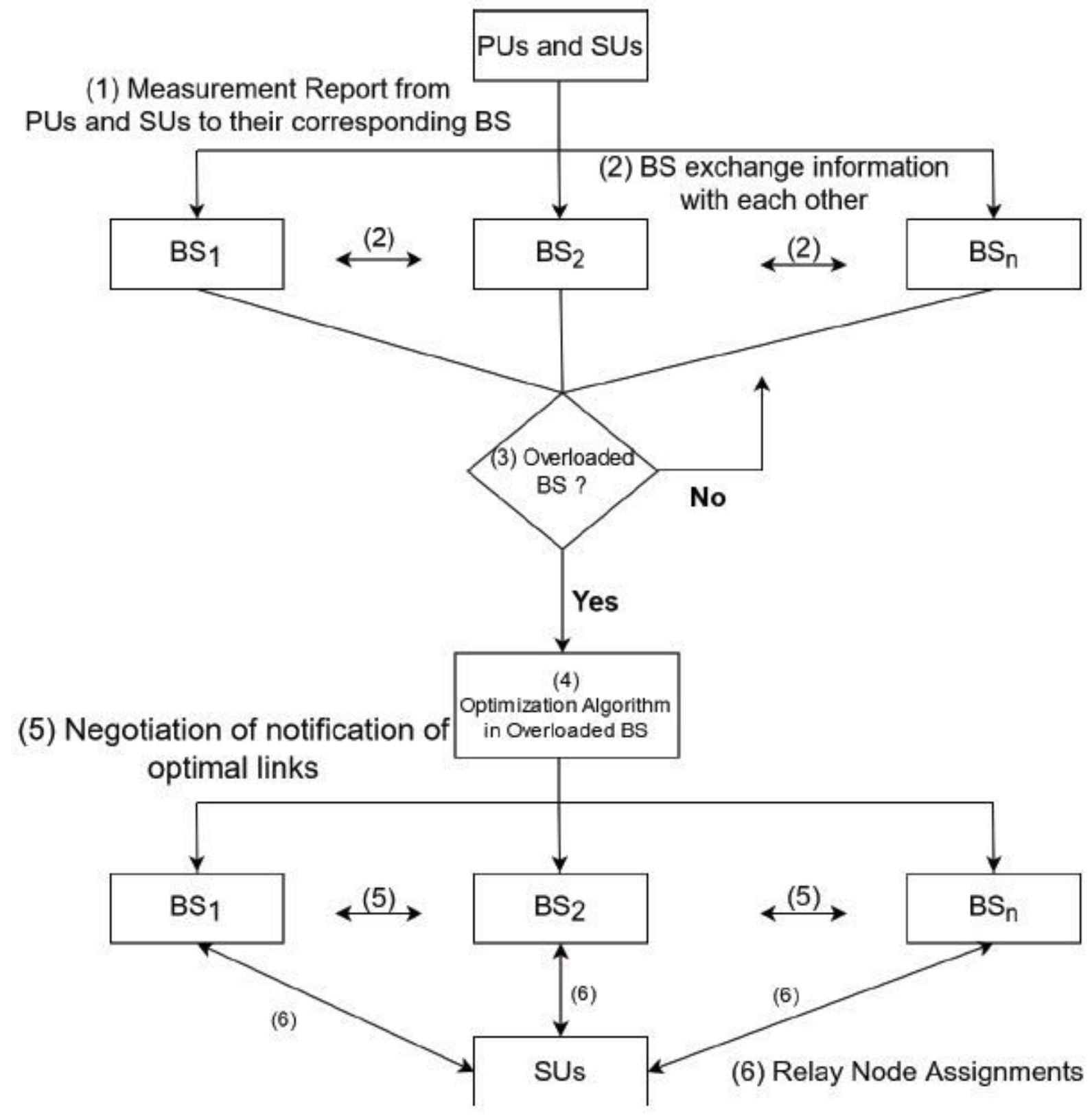

Figure 12

The message exchange flow between different components of the network 